\title{
NGC 6334 and NGC 6357
}

\section{Insights from spectroscopy of their OB star populations ${ }^{\star}$}

\author{
D. Russeil ${ }^{1}$, C. Adami ${ }^{1}$, J. C. Bouret ${ }^{1}$, A. Hervé ${ }^{2}$, Q. A. Parker ${ }^{3,4}$, A. Zavagno ${ }^{1}$, and F. Motte ${ }^{5}$ \\ 1 Aix Marseille Univ, CNRS, LAM, Laboratoire d'Astrophysique de Marseille, 13013 Marseille, France \\ e-mail: delphine.russeil@lam.fr \\ 2 Astronomical Institute ASCR, Fricova 298, 25165 Ondrejov, Czech Republic \\ 3 Department of Physics, Chong Yuet Ming Physics Building, The University of Hong Kong, Hong Kong \\ 4 Australian Astronomical Observatory, PO Box 915, North Ryde, NSW 1670, Australia \\ 5 Institut de Planétologie et d'Astrophysique de Grenoble (IPAG), Univ. Grenoble Alpes, CNRS/INSU, BP 53, \\ 38041 Grenoble Cedex 9, France
}

Received 10 October 2016 / Accepted 4 August 2017

\section{ABSTRACT}

\begin{abstract}
Aims. The formation of high-mass stars is still debated. For this reason, several projects such as Herschel-HOBYS are focussed on the study of the earliest phases of massive star formation. As a result, massive star-forming complexes such as NGC 6334 and NGC 6357 have been observed in the far-infrared to study their massive dense cores where massive stars are expected to form. However, to better characterise the environments of these cores we need to understand the previous massive star formation history. To better characterise the environment of these massive dense cores we study the previous high-mass star formation and how these stars act on their environments.

Methods. This study is based on the spectral classification of the OB stars identified towards NGC 6334 and NGC 6357 with spectra taken with the AAOmega spectrograph on the Anglo-Australian Telescope (AAT). From the subsequent spectral classification of 109 stars across these regions we were able to evaluate the following: distance, age, mass, global star-forming efficiency (SFE), and star formation rate (SFR) of the regions. The physical conditions of the ionised gas for both complexes was also derived.

Results. We confirm that NGC 6334 and NGC 6357 belong to the Saggitarius-Carina arm which, in this direction, extends from $1 \mathrm{kpc}$ to $2.2 \mathrm{kpc}$. From the location of the stars in Hertzprung-Russell diagram we show that stars older than $\sim 10 \mathrm{Myr}$ are broadly spread across these complexes, while younger stars are mainly located in the $\mathrm{H}$ II regions and stellar clusters. Our data also suggests that some of the young stars can be considered runaway stars. We evaluate a SFE of $0.019_{-0.007}^{+0.008}$ and $0.021_{-0.003}^{+0.004}$ and a SFR of $1.1 \times 10^{3} \pm$ $300 M_{\odot} \mathrm{Myr}^{-1}$ and $1.7 \times 10^{3} \pm 400 M_{\odot} \mathrm{Myr}^{-1}$ for NGC 6334 and NGC 6357, respectively. We note that 29 OB stars have X-ray counterparts, most of them belonging to NGC 6357. This suggests that molecular clouds in NGC 6357 are more impacted by X-ray flux and stellar winds than in NGC 6334. Finally, from the analysis of nebular lines (H $\alpha,[\mathrm{NII}]$, and [SII]) from spectra from several regions of ionised gas, we confirm that the filaments in NGC 6357 are shock heated.
\end{abstract}

Key words. stars: distances - HII regions - ISM: individual objects: NGC 6334 - ISM: individual objects: NGC 6357

\section{Introduction}

The H II regions NGC 6334 and NGC 6357 are two very active star-forming complexes in our Galaxy. They are apparently connected by a filamentary structure such that both regions may belong to a single overall complex (e.g. Russeil et al. 2010), even though they exhibit different morphologies. While NGC 6334 is the grouping of several optical H II regions located around a main molecular/dust ridge (Persi \& Tapia 2008), the morphology of NGC 6357 is dominated by a large cavity shaped by the massive open cluster Pismis 24 (e.g. Lortet et al. 1984) and also by a bright $\mathrm{H}$ II region $(\mathrm{G} 353.2+0.9)$ that traces a sharp boundary facing Pismis 24.

To study how massive stars form in NGC 6334 and NGC 6357 we need to determine the distance of the two regions and then place them in the current best model of the spiral arm

\footnotetext{
^ Full Tables 2 and A.1 and the normalised observed spectra displayed in Figs. B.1 are available at the CDS via anonymous ftp to cdsarc.u-strasbg. fr (130.79.128.5) or via

http://cdsarc.u-strasbg.fr/viz-bin/qcat?]/A+A/607/A86
}

structure in this direction in order to understand their relation to the surrounding molecular material and to estimate the star formation rates from the census of $\mathrm{OB}$ stars in each complex. In this paper we attempt to probe the recent massive star formation (as traced by O-B3 stars) for comparison with the present and likely future massive star formation as traced by the adjacent massive molecular cores and clumps (see Tigé et al. 2017; Russeil et al. 2010; Munoz et al. 2007).

As part of this program (Russeil et al. 2012, hereafter Paper I) observed 4 square degrees in and around NGC 6334, NGC 6357, and GM 1-24 in the $U, B$, and $V$ photometric bands with the VIMOS camera on the ESO-VLT. From the $U-B$ versus $B$ - $V$ colour-colour diagram 1302 candidate $\mathrm{O}$ to B3 stars with $V$ between 7.5 and 21 were found. The distances of these OB stars were then established assuming they are main-sequence stars. This hypothesis combined with their spectral types determined from the photometry resulted in a relative distance uncertainty of $\sim 50 \%$. This large stellar sample of such rare stars allowed us to carry out a valuable statistical study of the local structure of the Milky Way in this direction. The distribution of the stars showed 
Table 1. Summary details of our AAT 3.9 m - 2dF AAOmega spectroscopic observations.

\begin{tabular}{|c|c|c|c|c|c|c|c|}
\hline $\begin{array}{l}\text { Field centre } \\
\text { RA/Dec(J2000) }\end{array}$ & $\begin{array}{l}\text { Obs. date } \\
\text { (dd mm yy) }\end{array}$ & Arm & $\begin{array}{l}\text { Wavelength range } \\
(\AA)\end{array}$ & $\begin{array}{l}\text { Resolution } \\
F W H M(\AA)\end{array}$ & Gratings & $\begin{array}{l}\text { Exp. } \\
\text { (s) }\end{array}$ & $\begin{array}{l}\text { No. of } \\
\text { targets }\end{array}$ \\
\hline \multicolumn{8}{|c|}{ NGC 6334} \\
\hline $17^{\mathrm{h}} \cdot 19^{\mathrm{m}} \cdot 34.77^{\mathrm{s}}-35^{\circ} 49^{\prime} 59.30^{\prime \prime}$ & 14 & e/Red & $3358-5483 / 4$ & $3.5 / 5.3$ & $5 \mathrm{R}$ & 900 & 229 \\
\hline $17^{\mathrm{h}} 19^{\mathrm{m}} .34 .77^{\mathrm{s}}-35^{\circ} 49^{\prime} 59.30^{\prime \prime}$ & 03072014 & Blue/Red & $3358-5483 / 4955-8159$ & $3.5 / 5.3$ & $580 \mathrm{~V} / 385 \mathrm{R}$ & 5400 & 311 \\
\hline \multicolumn{8}{|c|}{ NGC 6357} \\
\hline $17 \mathrm{~h} 24 \mathrm{~m} \cdot 43.52$ & 030 & d & $3358-548$ & 3.5 & $85 \mathrm{R}$ & 900 & 160 \\
\hline $17 \mathrm{~h} 24^{\mathrm{m}} \cdot 43.52 \mathrm{~s}-34^{\circ} 28^{\prime} 05.00^{\prime \prime}$ & 03072014 & Blue/Red & $3358-5483 / 4955-8159$ & $3.5 / 5.3$ & $580 \mathrm{~V} / 385 \mathrm{R}$ & 5400 & 297 \\
\hline
\end{tabular}

peaks at 1.0, 1.8, 2.6, and $4.6 \mathrm{kpc}$ which were attributed to different spiral arms. The average distance of the NGC 6334 and NGC 6357 combined complex was found to be $1.75 \mathrm{kpc}$ which places it in the Sagittarius-Carina arm, although it appeared to be located slightly farther away than the first main stellar peak.

Distances to NGC 6334 and to NGC 6357 are still under debate. Recently, Lima et al. (2014) identified five embedded clusters within NGC 6357 and derived a distance of $1.78 \pm$ $0.1 \mathrm{kpc}$ (based on colour-magnitude diagrams), while Wu et al. (2014) and Chibueze et al. (2014) measured the parallax of $\mathrm{H}_{2} \mathrm{O}$ maser features associated with NGC 6334I(N) (a dense molecular clump associated with NGC 6334) and determined a distance of $1.35 \pm 0.15$ and $1.25 \pm 0.14 \mathrm{kpc}$, respectively, for NGC 6334 .

In this paper we present new spectroscopic observations taken with AAOmega fibre-spectroscopy system (Sharp et al. 2006) on the AAT for a sample of OB star candidates selected from Paper I. Our goal is to establish their spectral types and so determine new spectrophotometric distance of these stars to gain precision with respect to the photometric-only distance method previously used, which will then be used to refine the analysis of the NGC 6334 and NGC 6357 systems.

\section{Observations and data reduction}

We observed a sample of 874 stars extracted from the photometric catalogue of Paper I selected to have a $V$-band magnitude fainter than 14 mag. This sample was observed with the AAOmega spectrograph on the AAT in service mode on July 3 , 2014. AAOmega allows the simultaneous observation of up to 400 targets (including objects and sky positions) over a $2^{\circ}$ field with 2 arcsecond on-sky diameter fibres (Sharp et al. 2006). AAOmega is a dual beam system allowing both blue and red arm simultaneous observations; in this case the low-dispersion $580 \mathrm{~V}$ and $385 \mathrm{R}$ gratings were used. For a robust stellar spectral classification we principally needed to cover the spectral range from $3850 \AA$ to $5000 \AA$ because to classify O-B3 stars we need to identify some of the HeI and HeII lines at 4009, 4026, 4121, 4200, 4387, 4471, 4541, and $4686 \AA$. The grating central wavelengths were set to $4425 \AA$ for the blue arm and $6563 \AA$ ( $\mathrm{H} \alpha)$ for red arm. These gratings and settings deliver central spectral resolutions of $R=1253$ and $R=1234$, respectively. Given $2 \mathrm{dF}$ pointings were carefully selected to cover areas of each region centred on NGC 6334 and NGC 6357 that can sample the candidate O-B3 stars well. Target samples were further divided into two magnitude groups of $V=14$ to 17 and $V=18$ to 20 . This was done for observational efficiency by adapting exposure times to match the magnitude ranges of 15 min per $2 \mathrm{dF}$ field for the bright sample and 1 hour 30 min for the faint sample.
Standard spectral reduction was followed using the automated $2 \mathrm{dfDR}^{1}$ data reduction package with default values. The blue and red arm spectra were extracted, bias and flat-field corrected, wavelength calibrated, and sky subtracted. The blue and red part of the spectra were then combined to produce full wavelength range spectra taking care with the splicing to adjust continuum levels. We note that no flux calibration was attempted.

We placed a total of 997 fibres, 874 of which were placed towards the target stars while the others were placed towards stars, strategically located with respect to the H II regions and clusters. A first analysis of the 997 spectra allowed us to identify, and then exclude, 159 cool stars and 625 spectra exhibiting low signal-to-noise ratios $(\mathrm{S} / \mathrm{N})$ that prevented a decent spectral classification. In parallel, 104 fibres, placed in the direction of the faint stars, appeared to be fully dominated by nebular emission lines in both the blue and red spectra and were then used to probe basic ionised gas properties. Full details of the observations and instrumental configuration are given in Table 1.

\section{Spectral classification}

To assess spectral type for each stellar spectrum we compared our data to the spectral atlas of Maíz Apellániz et al. (2016) (O stars) and Walborn \& Fitzpatrick (1990) (B stars), both of which provide medium-resolution spectra $(R=2500$ and 2933, respectively) in the 4000 to $4900 \AA$ range. After degrading the template spectra to our spectral resolution $(R=1253)$ we performed the fitting over the key 4000-4900 $\AA$ range between the normalised observed spectra and the templates (see Figs. B.1).

For most observed stars with $V>17$ the $\mathrm{S} / \mathrm{N}$ in the blue was often too low to perform a reliable classification. However, for 11 stars where the blue spectra were inadequate we were able to use the red part of the spectrum instead following the qualitative approach of Walborn (1980). With this approach it is not possible to determine the luminosity class, so dwarf class was assumed. In the red spectra, O-type stars are identified from the ratio of He I $\lambda 5876 \AA$ to He II $\lambda 5411 \AA$, which passes through unity at type $\mathrm{O} 7$, while He I $\lambda 5876 \AA$ is absent at type O3. The O III $\lambda 5592 \AA$ is visible up to O9.5 V spectrum, while He II $\lambda 5411 \AA$ persists until B0.2V. At type B0.5 V, Si III $\lambda 5740 \AA$ becomes visible and remains so through to type B1.5V. Finally, B2.5 V to B3 V spectra are identified from the Si II+Mg II $\lambda 6347 \AA$ visibility.

Ultimately, 109 stars were classified as hot (O to B3) stars (see Table 2). Their spectra are displayed in Figs. B.1 and

1 http://WWW . aao.gov . au/science/software/2dfdr 
Table 2. The first ten entries of the $109 \mathrm{O}$ to $\mathrm{B} 3$ star sample.

\begin{tabular}{|c|c|c|c|c|c|c|c|c|c|c|c|}
\hline No. ${ }^{a}$ & $\begin{array}{l}\text { RA } \\
(\mathrm{J} 2000)\end{array}$ & $\begin{array}{l}\text { Dec } \\
(\mathrm{J} 2000)\end{array}$ & $\begin{array}{l}\text { Spec. } \\
\text { Type }^{b}\end{array}$ & $\begin{array}{c}V^{c} \\
\operatorname{mag}\end{array}$ & $\begin{array}{c}B^{c} \\
\mathrm{mag}\end{array}$ & $\begin{array}{c}U^{c} \\
\mathrm{mag}\end{array}$ & $\begin{array}{c}A_{V} \\
\mathrm{mag}\end{array}$ & $\begin{array}{c}d \\
\mathrm{kpc}\end{array}$ & $\begin{array}{c}M \\
M_{\odot} \\
\end{array}$ & $\begin{array}{c}\log (\text { age }) \\
y r\end{array}$ & $\begin{array}{l}\text { 2MASS } \\
\text { designation }\end{array}$ \\
\hline 1 & $17: 16: 03.8$ & $-36: 18: 51.8$ & $\mathrm{~B} 2 \mathrm{~V}$ & $15.682 \pm 0.009$ & $17.596 \pm 0.019$ & $18.212 \pm 0.062$ & $8.40 \pm 0.30$ & $0.90 \pm 0.30$ & 8.6 & 7.3 & $17160382-3618517$ \\
\hline 2 & $17: 16: 04.3$ & $-36: 16: 55.2$ & $\mathrm{~B} 2 \mathrm{~V}$ & $15.880 \pm 0.009$ & $17.597 \pm 0.019$ & $18.056 \pm 0.058$ & $7.71 \pm 0.29$ & $1.88 \pm 0.81$ & 11.8 & 7.1 & $17160431-3616548$ \\
\hline 3 & $17: 16: 29.5$ & $-36: 20: 56.2$ & B3 & $16.286 \pm 0.011$ & $18.057 \pm 0.023$ & $18.554 \pm 0.067$ & $7.69 \pm 0.31$ & $1.11 \pm 0.82$ & 6.4 & 7.6 & $17162955-3620558$ \\
\hline 4 & $17: 16: 35.0$ & $-36: 27: 36.7$ & $\mathrm{~B} 2 \mathrm{~V}$ & $15.168 \pm 0.006$ & $16.530 \pm 0.010$ & $16.804 \pm 0.029$ & $6.25 \pm 0.21$ & $1.91 \pm 0.64$ & 8.6 & 7.3 & $17163510-3627367$ \\
\hline 5 & $17: 16: 53.5$ & $-36: 19: 22.4$ & B2 V & $15.534 \pm 0.008$ & $16.909 \pm 0.014$ & $17.215 \pm 0.036$ & $6.30 \pm 0.23$ & $2.20 \pm 0.74$ & 8.6 & 7.3 & $17165359-3619225$ \\
\hline 6 & $17: 17: 00.5$ & $-36: 13: 36.0$ & B3 Ia & $14.887 \pm 0.006$ & $17.285 \pm 0.015$ & $18.304 \pm 0.052$ & $9.82 \pm 0.31$ & $2.64 \pm 1.12$ & 24.5 & 6.8 & $17170051-3613362$ \\
\hline 7 & $17: 17: 01.4$ & $-36: 22: 01.6$ & B2 V & $15.988 \pm 0.010$ & $17.713 \pm 0.021$ & $18.209 \pm 0.063$ & $7.66 \pm 0.30$ & $1.45 \pm 0.48$ & 8.6 & 7.3 & $17170147-3622014$ \\
\hline 8 & $17: 17: 02.4$ & $-36: 20: 05.0$ & $\mathrm{~B} 2 \mathrm{~V}$ & $15.657 \pm 0.009$ & $17.044 \pm 0.015$ & $17.432 \pm 0.044$ & $6.35 \pm 0.24$ & $2.28 \pm 0.76$ & 8.6 & 7.3 & $17170247-3620049$ \\
\hline 9 & $17: 17: 11.4$ & $-36: 19: 39.5$ & B2 V & $16.083 \pm 0.010$ & $17.494 \pm 0.018$ & $17.900 \pm 0.055$ & $6.44 \pm 0.26$ & $2.66 \pm 0.89$ & 8.6 & 7.3 & $17171143-3619393$ \\
\hline $\begin{array}{l}10 \\
\text { etc... }\end{array}$ & $17: 17: 18.5$ & $-36: 21: 38.6$ & B3 V & $14.846 \pm 0.006$ & $16.084 \pm 0.010$ & $16.417 \pm 0.028$ & $5.61 \pm 0.19$ & $1.49 \pm 0.60$ & 6.4 & 7.6 & $17171858-3621384$ \\
\hline
\end{tabular}

Notes. Full table is available at the CDS. ${ }^{(a)}$ Stars with an X-Ray counterpart are indicated with an asterisk ; ${ }^{(b)}$ stars with no luminosity class are classified from the red part of their spectrum (we assume they are dwarf stars); ${ }^{(c)} U B V$ photometry is from Russeil et al. (2012).

their alternative names (from the SIMBAD database) and crossidentification with 2MASS and Gaia-DR1 (Lindegren et al. 2016) catalogues are presented Table A.1. A visual inspection of the spectra was performed to check and precise the classification following the qualifiers listed in Table 3 of Sota et al. (2011). Because of our low resolution it is difficult to identify binary stars ${ }^{2}$ or to specify any spectral peculiarity. Indeed, spectral peculiarity or binarity can modify the luminosity and then impact the distance estimate. For example, Walborn et al. (2014) note that the $V z$ characteristic corresponds to lower luminosity. In this frame we checked our star sample and find that no star fulfils the $V z$ criterion (Arias et al. 2016) or exhibits detectable nitrogen, silicon, or carbon emission features. Comparing the spectroscopic classification of this hot star subsample with the photometric equivalent from Paper I, we note that $27 \%$ have less than one type of difference, $23 \%$ have one or one and half types of difference, while the others have a greater number of differences (four type differences on average). In addition we note that $93 \%$ of the sample are B stars (which becomes $84 \%$ when adding the bright $\mathrm{OB}$ stars from the literature).

\section{Discussion}

\subsection{Stellar distance}

For the newly classified O to B3 stars we determine their distances and the extinction (see Table 2) by combining their present spectral types with their $B$ and $V$ photometry established in Paper I and using the extinction relation $A_{V}=R_{V} \times$ $E(B-V)$, where $R_{V}$ and $E(B-V)$ are band integrated quantities (Cardelli et al. 1989). To this end, we applied the traditional spectrophotometric method and used the absolute magnitude $M_{V}$ and intrinsic colour calibrations (Schmidt-Kaler 1983; Humphreys \& McElroy 1984; Vacca et al. 1996; Sung et al. 2013), while the adequat $R_{V}$ value is taken from Russeil et al. (2012). The uncertainty on the distance takes into account the uncertainties from the photometry, the $R_{V}$ and the calibration (adopting one class, and one spectral sub-type uncertainty). Comparing the photometric and spectroscopic classifications of the spectral types allows us to show that the more accurate spectroscopic classification results in a statistical decrease in distance

\footnotetext{
2 To identify possible binarity we used the "duplicate" flag from the Gaia-DR1 catalogue, the photometric comparison between the Gaia $G$-band and $V$-band (Paper I) magnitudes, and the "prox" flag from the 2MASS point source catalogue and find that only the stars $30,32,49$, 72 , and 82 could be binary or multiple stellar systems (see Appendix A)
}

of about $41 \%$. The O-B3 star distances distribution is shown Fig. 2-upper panel. We complemented our sample with 25 stars belonging to NGC 6357 (14 stars) and NGC 6334 (11 stars), for which the spectral type (deduced from spectral analysis or $U B V$ $\mathrm{H} \beta$ photometry) and photometry were retrieved from the literature (see Table A.2).

The distance histogram clearly shows a peak at around $1.7 \mathrm{kpc}$ (with a sub-peak at $1.1 \mathrm{kpc}$ ), and two other features at around 2.3 and $2.7 \mathrm{kpc}$. Similarly, at $l=314^{\circ}$ Galactic line of sight, Carraro (2011) reported the detection of two different groups of young field stars at 1.5 and $2.5 \mathrm{kpc}$. This is in agreement with our previous result (cf. Fig. 7 in Russeil et al. 2012). From the Hou \& Han (2014) Milky Way spiral arm model, the line of sight in the direction of the overall NGC 6334 NGC 6357 complex is supposed to cross the Sagittarius-Carina, Scutum-Crux, and Norma spiral arms at distances $\sim 0.9, \sim 3$, and $\sim 4.5 \mathrm{kpc}$, respectively, while in the Russeil et al. (2007) model it is supposed to cross these arms at slightly different distances of $\sim 1.5, \sim 3$, and $\sim 4.3 \mathrm{kpc}$ (mainly because of the departure of the Sagittarius-Carina arm with respect to a logarithmic spiral model in this direction). Consequently, the 2.3 to $2.7 \mathrm{kpc}$ peak can be assigned to the Scutum-Crux arm, while the first peak is assigned to the Sagittarius-Carina arm.

To refine the spiral arm distances, we collected from the literature star clusters known in the direction of NGC 6334 and NGC 6357 (Fig. A.1) and for which the distances have been determined independently of any velocity or association (see Table 3). The distance and reddening are either from stellar spectrophotometric information or from isochrone fitting to the observed colour-magnitude distributions. For a broader context, we also collected, from Kharchenko et al. (2013), clusters within a $3.7^{\circ}$ radius centred at $l, b=351.33^{\circ}, 0.07^{\circ}$, covering both NGC 6334 and NGC 6357 comprising a total of 39 clusters. The distance histogram for these clusters is shown in Fig. 2-lower panel. It shows mainly a broad distribution peaking at $\sim 1.2 \mathrm{kpc}$ with a proportion of young clusters increasing with distance. The clusters exciting the H II regions in NGC 6334 and NGC 6357 belong mainly to the youngest and farthest part of the complex between 1.6 and $2.1 \mathrm{kpc}$. The cluster ages distribution suggests a possible stellar age gradient across the Carina arm. This gradient was already reported by Melnik et al. (1998).

At higher Galactic latitude we note the $\mathrm{H} \mathrm{II}$ region Sh2-10 $\left(l, b=352.44^{\circ},+2.26^{\circ} ; \alpha, \delta=17 \mathrm{~h} 17 \mathrm{~m} 11.43 \mathrm{~s}\right.$, $\left.-34^{\circ} 03^{\prime} 07.6294^{\prime \prime}\right)$. It is excited by the SCO-OB4 association at a distance of $1.1 \mathrm{kpc}$ (Kharchenko et al. 2005) similar to the distance of cluster Bochum 13 located to the north of NGC 6334 


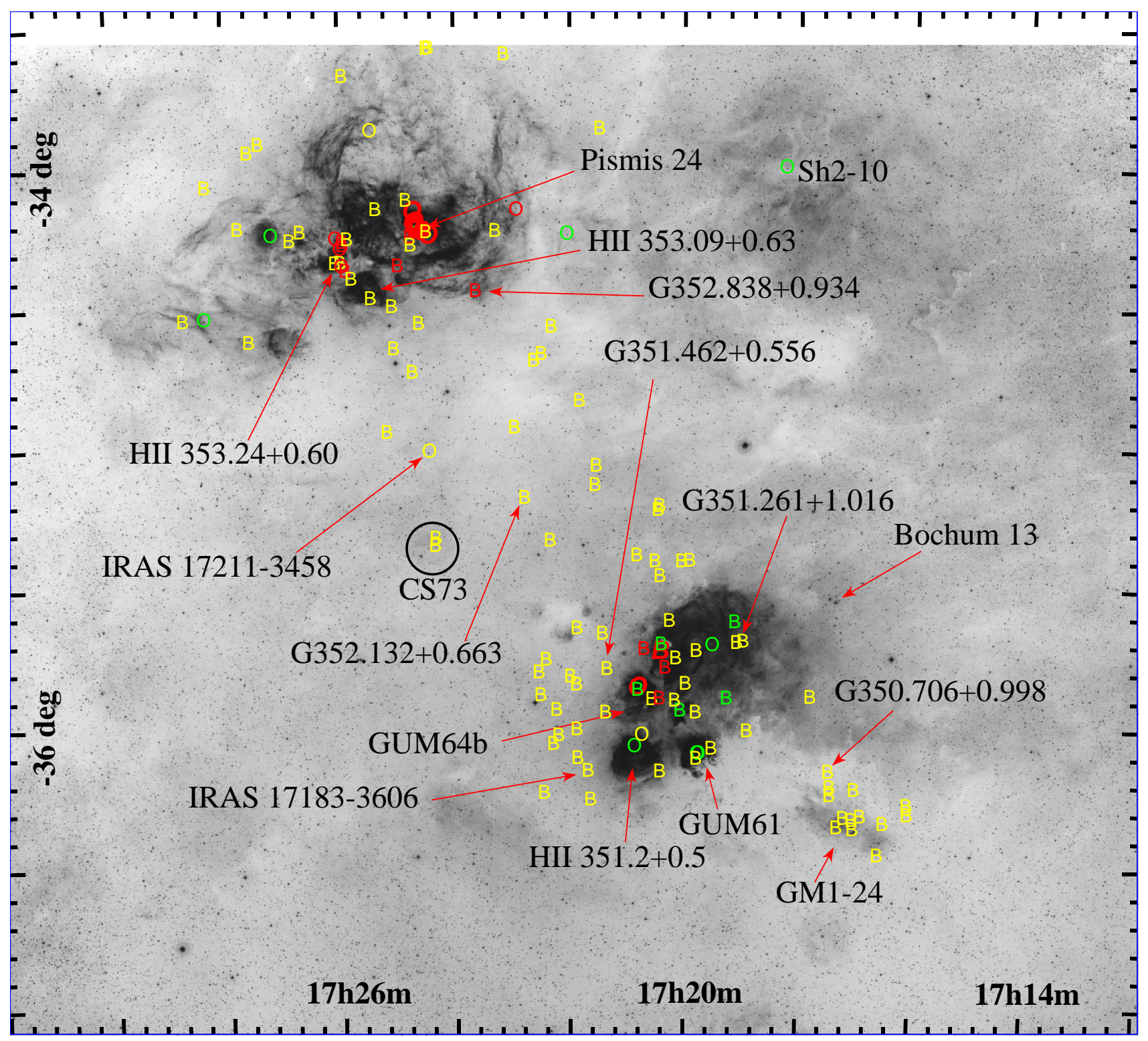

Fig. 1. Global $\mathrm{H} \alpha$ view (AAO/UKST H $\alpha$ image, Parker et al. 2005) of NGC 6334 and NGC 6357. The position of the objects discussed in the text are indicated. The sample of O to B3 stars are overlaid (labelled "O" and "B"). Red symbols indicate stars with X-ray counterparts. The green and bold red symbols are stars from the literature.

(see distance listed in Table 3). Both H II regions can be assigned to the closest identified stellar peak $(1.1 \mathrm{kpc})$.

\subsection{Distance to particular regions}

Several of our newly identified and sampled O-B3 stars are found in the direction of particular regions (Fig. 1), which therefore allow us to constrain their distances.

Pismis $24\left(\alpha, \delta=17 \mathrm{~h} 24 \mathrm{~m} 38.4 \mathrm{~s},-34^{\circ} 12^{\prime} 49^{\prime \prime}\right)$ : four stars are located towards Pismis 24 from which we determine a mean distance of $2.46 \pm 1.44 \mathrm{kpc}$. This distance is in agreement with the value established by Massey et al. (2001).

$H I I$ region $353.24+0.60(\alpha, \delta=17 \mathrm{~h} 26 \mathrm{~m} 06.12 \mathrm{~s}$, $\left.-34^{\circ} 18^{\prime} 16.74^{\prime \prime}\right)$ : toward this $\mathrm{H}$ II region, located in the NGC 6357 star-forming complex, a cluster of young stellar objects was described by Fang et al. (2012) and named the "B cluster" more recently by Massi et al. (2015). Towards this cluster we note four OB stars giving a distance of $2.37 \pm$ $0.81 \mathrm{kpc}$.

G352.838+0.934 $\left(\alpha, \delta=17 \mathrm{~h} 23 \mathrm{~m} 40.11 \mathrm{~s},-34^{\circ} 26^{\prime} 58.59^{\prime \prime}\right)$ : listed as a 2.85 arcmin radius $\mathrm{H}$ II region by Anderson et al. (2014) and associated with a $\sim 2$ arcmin radius infrared bubble (MWP1G352835+009401) by Simpson et al. (2012), it has a previously assigned distance determined by assuming a spatial grouping with NGC 6357. It exhibits clear WISE-22 $\mu \mathrm{m}$ emission in its central part and exhibits a semi-arc Spitzer $-8 \mu \mathrm{m}$ photodissociation region (PDR) with pillars pointing towards an B0Iab star, for which we establish a distance of $4.59 \pm 2.52 \mathrm{kpc}$. However, the spectral type of the star (star 69) is quite uncertain due to the poor $\mathrm{S} / \mathrm{N}$ (Fig. B.1).

CS73 ( $\left.\alpha, \delta=17 \mathrm{~h} 24 \mathrm{~m} 24.62 \mathrm{~s},-35^{\circ} 21^{\prime} 25.45^{\prime \prime}\right)$ : in the direction of the bubble $\operatorname{CS} 73\left(l, b=352.174^{\circ},+0.297^{\circ}\right)$, which has a reported average bubble radius of $\sim 5.5$ arcmin identified in the mid-infrared by Churchwell et al. (2007), we find two 
Table 3. Cluster summary.

\begin{tabular}{|c|c|c|c|c|c|c|c|}
\hline Cluster & $\begin{array}{l}\text { Coordinates } \\
\text { J2000 }\end{array}$ & $\begin{array}{l}\text { Assoc. } \\
\text { region }\end{array}$ & $\begin{array}{c}\text { Distance } \\
\mathrm{kpc}\end{array}$ & $\begin{array}{c}\log (\text { age }) \\
y r\end{array}$ & $E(B-V)$ & $\begin{array}{l}\text { Radius } \\
\text { arcmin. }\end{array}$ & Reference \\
\hline \multicolumn{8}{|c|}{ NGC 6334} \\
\hline \multirow[t]{2}{*}{ Bochum 13} & $17^{\mathrm{h}} \cdot 17^{\mathrm{m}} \cdot 24.0^{\mathrm{s}}-35^{\circ} 33^{\prime} 00^{\prime \prime}$ & & 1.34 & 7.25 & 0.82 & 9.30 & 1 \\
\hline & & & 1.08 & 6.82 & & 7 & 3 \\
\hline MWSC2558 & $17^{\mathrm{h}} .17^{\mathrm{m}} .14 .6^{\mathrm{s}}-36^{\circ} 20^{\prime} 07^{\prime \prime}$ & GM1-24 & 2.02 & 6.00 & 1.46 & 5.40 & 1 \\
\hline MWSC2577 & $17^{\mathrm{h}} .20^{\mathrm{m}} \cdot 48.6^{\mathrm{s}}-36^{\circ} 06^{\prime} 57^{\prime \prime}$ & $\mathrm{G} 351.2+0.5$ & 1.13 & 8.32 & 1.03 & 14.7 & 1 \\
\hline \multirow[t]{2}{*}{ MWSC2575 } & $17^{\mathrm{h}} \cdot 20^{\mathrm{m}} \cdot 38.8 \mathrm{~s}-35^{\circ} 52^{\prime} 59^{\prime \prime}$ & GUM64b & 0.89 & 8.45 & 0.75 & 4.20 & 1 \\
\hline & & NGC 6357 & & & & & \\
\hline \multirow[t]{4}{*}{ Pismis24 } & $17^{\mathrm{h}} \cdot 24^{\mathrm{m}} \cdot 38.4^{\mathrm{s}}-34^{\circ} 12^{\prime} 49^{\prime \prime}$ & $\mathrm{G} 353.2+0.9$ & 1.77 & 6.00 & 2.19 & 6.6 & 1 \\
\hline & & & 2.0 & 6.69 & 1.75 & 1.5 & 2 \\
\hline & & & 1.7 & 6.0 & 1.87 & & 4 \\
\hline & & & 2.5 & 6.24 & 1.73 & 2.7 & 5 \\
\hline VVVCL167 & $17^{\mathrm{h}} \cdot 25^{\mathrm{m}} \cdot 09.0^{\mathrm{s}}-34^{\circ} 11^{\prime} 13^{\prime \prime}$ & & 1.6 & 6.95 & 1.47 & 0.6 & 2 \\
\hline \multirow[t]{2}{*}{ AH03J1725-34.4 } & $17^{\mathrm{h}} \cdot 25^{\mathrm{m}} \cdot 32.0^{\mathrm{s}}-34^{\circ} 24^{\prime} 20^{\prime \prime}$ & H II $353.09+0.63$ & 1.48 & 6.0 & 2.63 & 5.4 & 1 \\
\hline & & & 1.9 & 6.69 & 1.99 & 1.7 & 2 \\
\hline BDS101 & $17^{\mathrm{h}} \cdot 25^{\mathrm{m}} \cdot 34.0^{\mathrm{s}}-34^{\circ} 23^{\prime} 09^{\prime \prime}$ & H II $353.09+0.63$ & 1.6 & 6.69 & 1.95 & 0.7 & 2 \\
\hline MWSC2598 & $17.26^{\mathrm{m}} \cdot 49.9^{\mathrm{s}}-34^{\circ} 40^{\prime} 59^{\prime \prime}$ & & 0.82 & 7.95 & 0.75 & 1 & 1 \\
\hline
\end{tabular}

References. 1 = Kharchenko et al. (2013) and Kharchenko et al. (2016); 2 = Lima et al. (2014); 3 = Morales et al. (2013); 4 = Fang et al. (2012); 5 = Massey et al. (2001).

B-type stars with distances of $1.56 \pm 0.98$ and $2.23 \pm 0.92 \mathrm{kpc}$, respectively. The latter, of $\mathrm{B} 2.5 \mathrm{~V}$ type, is more centrally located within the bubble and so suggests a distance of about $2 \mathrm{kpc}$ for the bubble itself. In addition, faint but clear $\mathrm{H} \alpha$ emission fills the bubble suggesting it is a $\mathrm{H}$ II region located in the Sagittarius spiral arm but in a low-density area.

IRAS 17211-3458 ( $\left.\alpha, \delta=17 \mathrm{~h} 24 \mathrm{~m} 27.6 \mathrm{~s},-35^{\circ} 01^{\prime} 02^{\prime \prime}\right)$ : we find an $09 \mathrm{~V}$ star $(d=1.37 \pm 0.32 \mathrm{kpc})$ centred on this IRAS source $\left(l, b=351.474^{\circ},+00.549^{\circ}\right)$, which was classified as an ultra-compact $\mathrm{H}$ II region by Bronfman et al. (1996). No $\mathrm{H} \alpha$ emission is observed, while at Spitzer-8 $\mu \mathrm{m}$ only a faint diffuse emission $(\sim 1.7$ arcmin across $)$ is noted around the star.

$G 352.132+0.663\left(\alpha, \delta=17 \mathrm{~h} 22 \mathrm{~m} 48.84 \mathrm{~s},-35^{\circ} 11^{\prime} 06.53^{\prime \prime}\right)$ : this was identified as a candidate $\mathrm{H}$ II region by Anderson et al. (2014). We identify a B3 star in its direction. However, we were not able to assign a luminosity class for this star. Assuming it is a main-sequence star, this gives a distance of $0.89 \pm 0.66 \mathrm{kpc}$. This distance increases to $1.5 \pm 0.67 \mathrm{kpc}$ if it is a class III star. G351.462+0.556 $\left(\alpha, \delta=17 \mathrm{~h} 21 \mathrm{~m} 22.70 \mathrm{~s},-35^{\circ} 47^{\prime} 50.79^{\prime \prime}\right)$ : this region is listed by Anderson et al. (2014) as a radio quiet $\mathrm{H}$ II region and it has a small $\mathrm{H} \alpha$ counterpart with a Vlsr of $-8 \mathrm{~km} \mathrm{~s}^{-1}$ (Russeil et al. 2016). Based on its mid-infrared morphology this region was classified as a possible bow-shock due to a runaway star (Russeil et al. 2016). Here we identify a B1 III star with a distance of $2.46 \pm 1.06 \mathrm{kpc}$.

G351.261+01.016 ( $\left.\alpha, \delta=17 \mathrm{~h} 18 \mathrm{~m} 57.122 \mathrm{~s},-35^{\circ} 41^{\prime} 57.54^{\prime \prime}\right)$ : in the direction of this region (Anderson et al. 2014) we find a B2 V star $(d=4.11 \pm 1.37 \mathrm{kpc})$. The radio continuum emission $\left(\mathrm{S}_{9 \mathrm{GHz}}=310 \mathrm{mJy}\right)$ estimated by Anderson et al. (2011) corresponds to a O9.5/B0 V star (based on Panagia 1973) suggesting that the star we identify is not alone in exciting the region. This is in agreement with its belonging to the cluster [BDS2003]99 (Morales et al. 2013). The radio recombination Vlsr of the region is $-3.72 \mathrm{~km} \mathrm{~s}^{-1}$ (given by Wenger et al. 2013), but the $\mathrm{H} \alpha$ velocity is measured around $-11 \mathrm{~km} \mathrm{~s}^{-1}$ (Russeil et al. 2016) suggesting that either the region is not linked to NGC 6334 or it has some internal motion. If the distance of the star and the $\mathrm{H} \alpha$ velocity suggest the region is not linked to NGC 6334, the radio velocity and the morphological correlation between the dust emission (Spitzer-8 $\mu \mathrm{m})$ and the optical $(\mathrm{H} \alpha)$ extinction feature suggest the opposite. For this region we expect that Gaia will allow us to solve the problem.

IRAS 17183-3606 ( $\left.\alpha, \delta=17 \mathrm{~h} 21 \mathrm{~m} 43.02 \mathrm{~s},-36^{\circ} 09^{\prime} 33.68^{\prime \prime}\right)$ : we find a B0 V star $(d=0.93 \pm 0.30 \mathrm{kpc})$ centred on this IRAS source $\left(l, b=351.203^{\circ},+00.293^{\circ}\right)$. It is clearly seen as an extended but patchy source in WISE- $22 \mu \mathrm{m}$ and Spitzer- $24 \mu \mathrm{m}$ images with a size of $\sim 2 \times 4$ arcmin. It appears as a narrow and small filament at Spitzer-8 $\mu \mathrm{m}$. Despite its distance, no $\mathrm{H} \alpha$ and radio emission is observed.

G350.706+0.998 ( $\left.\alpha, \delta=17 \mathrm{~h} 17 \mathrm{~m} 26.75 \mathrm{~s},-36^{\circ} 09^{\prime} 47.92^{\prime \prime}\right)$ : in the centre of this region (Anderson et al. 2014), also identified as the mid-infrared bubble CS98 (Churchwell et al. 2007), we find a B1V star with a distance of $1.10 \pm 0.48 \mathrm{kpc}$. A bow-shock feature is also clearly seen at WISE-22 $\mu \mathrm{m}$ and Herschel-70 $\mu \mathrm{m}$, underlining the asymmetry of the bubble. This feature can be produced by a runaway star moving at high relative velocity (Gvaramadze et al. 2011) or by a stellar wind within the $\mathrm{H}$ II region associated with such a rapidly moving star (Mackey et al. 2016). Because the WISE-22 $\mu \mathrm{m}$ arc is well inside the Spitzer- $8 \mu \mathrm{m}$ bubble we favour the second hypothesis. However, this region is not seen in $\mathrm{H} \alpha$, probably because of the strong extinction in front of it.

GM 1-24 ( $\left.\alpha, \delta=17 \mathrm{~h} 17 \mathrm{~m} 04.0 \mathrm{~s},-36^{\circ} 21^{\prime} 14^{\prime \prime}\right)$ : several stars are observed in the direction of GM 1-24 and its adjacent dark area. A mean distance of $1.58 \pm 0.62 \mathrm{kpc}$ is found from stars towards the dark area, while stars observed toward the GM 1-24 $\mathrm{H} \alpha$ emission span from 1.44 to $2.66 \mathrm{kpc}$. In the direction of the radio source $\mathrm{G} 350.5+0.95$ located at the centre of the mid-infrared bubble CS103 (Churchwell et al. 2007), a B2 V star at $1.45 \pm 0.48 \mathrm{kpc}$ is observed. 

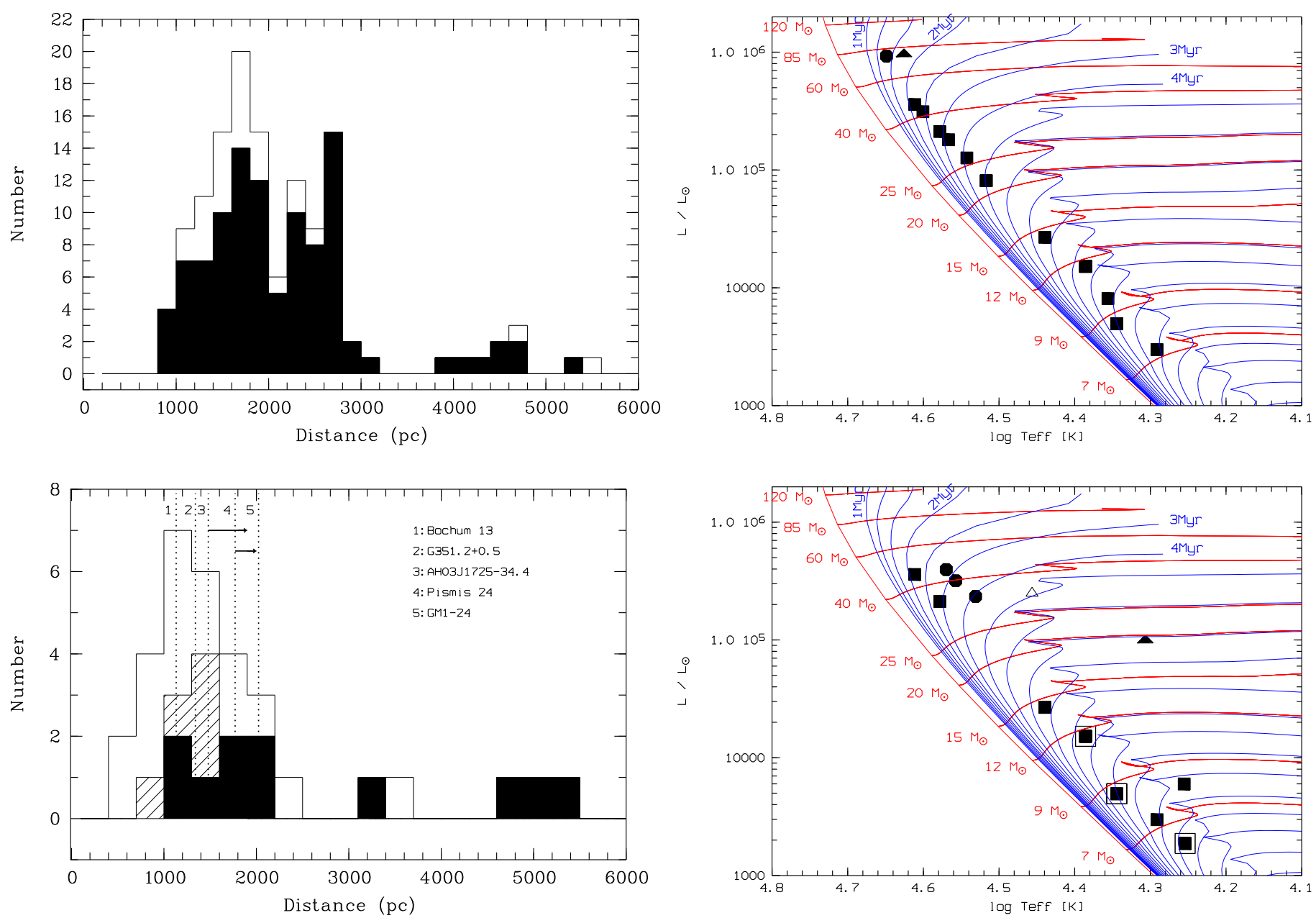

Fig. 2. Upper panel: distribution of O-B3 stars distances. The black histogram corresponds to the present O-B3 sample, while the full white histogram includes the O-B3 stars collected from the literature. Lower panel: distribution of stellar clusters collected from the literature (white, hashed, and black histograms correspond to all clusters, clusters with $10<$ age $<100 \mathrm{Myr}$, and clusters with age $\leq 10 \mathrm{Myr}$, respectively).

\subsection{Hertzprung-Russell diagram: star formation efficiency and rate}

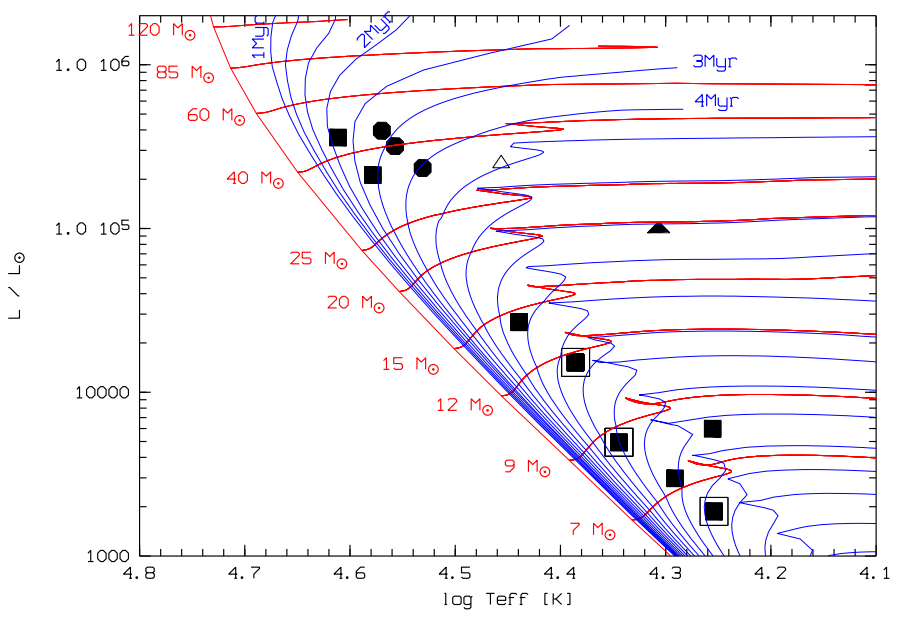

Fig. 3. HR diagram for NGC 6357 (upper panel) and NGC 6334 (lower panel). Filled squares, circles, and triangles identify stars of luminosity classes IV and V, giants (classes II and III), and supergiants (class I), respectively. Open symbols in the lower panel are for GM1-24. The blue and red curves are Geneva isochrones and evolutionary tracks, respectively.

calibrations) for O- and B-type stars, respectively. Similarly, from the $V$-band photometry and distance uncertainties we estimated $\sim 0.32 \mathrm{dex}$ and $\sim 0.45 \mathrm{dex}$ in $\log L / L_{\odot}$ (with the adopted $\mathrm{BC}$ calibration) for O- and B-type stars.

From the previous section we assumed that stars between In order to derive the star formation rate (SFR) and star for tion efficiency (SFE) we need to determine stellar masses and ages. To do this, we placed the stars on the Hertzprung-Russell (HR) diagram and compared their positions with the Geneva stellar isochrones and evolutionary tracks ${ }^{3}$ (Lejeune \& Schaerer 2001) computed for solar metallicity and no rotation. The star bolometric luminosities were calculated using the observed $V$ magnitudes (Russeil et al. 2012) and the spectral types, $A_{V}$ extinctions and distances determined in this paper. For that, following Wright et al. (2015), the bolometric corrections (BC) were taken from Martins \& Plez (2006) for O-type stars and from Crowther et al. (2006) and Humphreys \& McElroy (1984) for B-type stars. The effective temperatures were assigned as a function of the spectral type using the tabulations of Martins et al. (2005) for O-type stars and Trundle et al. (2007) for B-type stars. Given the typical uncertainties of half a type in the spectral classification and one class in luminosity, we evaluated uncertainties of $\sim 0.04$ dex and $\sim 0.07 \operatorname{dex}$ in $\log T_{\text {eff }}$ (from the adopted 3 Downloaded from the interactive tools web page: http://obswww.
unige.ch/Recherche/evol/

1.1 and $2.3 \mathrm{kpc}$ belong to the NGC 6334 and NGC 6357 starforming complexes. HR diagrams were produced separately for stars in the direction of NGC 6334 (and GM1-24) and NGC 6357 (Fig. 3). Stars with low $\mathrm{S} / \mathrm{N}$ were not used in these plots. For every star, we estimated the age and the initial mass by minimising the difference between the Bolometric Luminosity and the temperature with the values from the Geneva modelled evolutionary tracks (Lejeune \& Schaerer 2001). We then were able to look at the age and spatial distribution of the stars (Fig. 4). The typical uncertainties are $18 \%$ on the mass and $37 \%$ and $94 \%$ on the age for O- and B-type stars, respectively.

From Fig. 3 we note a large age spread among the members for both regions suggesting the star formation has been in progress for at least $5 \mathrm{Myr}$, while a stellar population older than $12.6 \mathrm{Myr}(\log ($ age $)=7.1)$ is also noted. Inversely, stars as young as $1.3 \mathrm{Myr}$ are also observed in NGC 6357. The old population is spatially spread (Fig. 4) throughout the field, including the area between the two main regions. In NGC 6334 stars younger than 


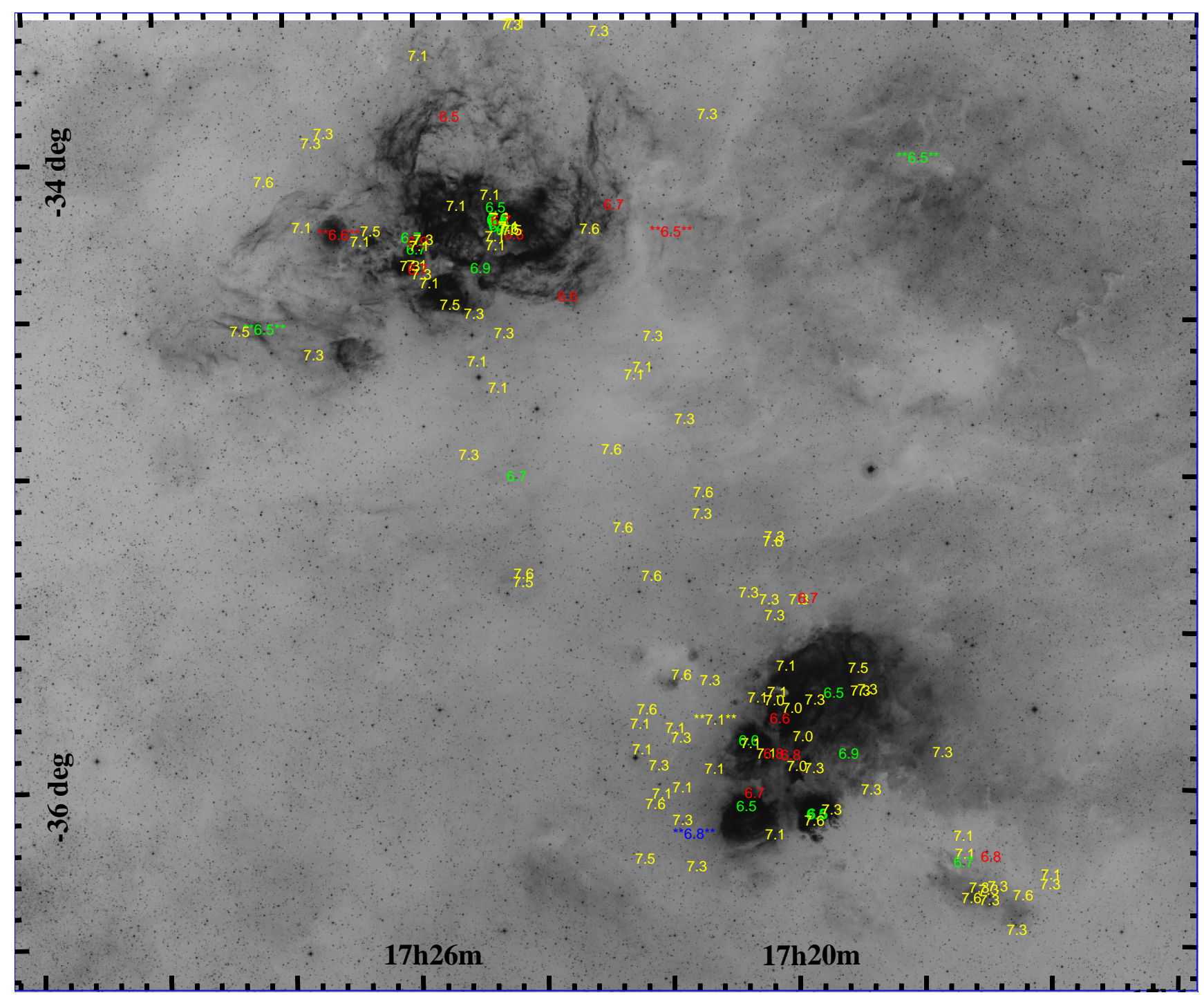

Fig. 4. Age $(\log ($ age $))$ of stars overlaid on the $\mathrm{H} \alpha$ view (AAO/UKST H $\alpha$ image, Parker et al. 2005) of NGC 6334 and NGC 6357 . Values between double-star marks identify possible runaway stars (see text). Values in green, red, and blue mark stars with $\log ($ age) $<7$ with distances $1 \leq d \leq$ $2.3 \mathrm{kpc}, d>2.3 \mathrm{kpc}, d<1 \mathrm{kpc}$, respectively.

$10 \mathrm{Myr}$ are mainly located in the $\mathrm{H}$ II regions and give an age between 1.9 and 3.9 Myr. GM1-24 seems not to have formed stars for the last $5 \mathrm{Myr}$.

In NGC 6357, stars younger than $5 \mathrm{Myr}$ can be split into a $~ 1.4$ Myr (2 stars) and 4.6 Myr (13 stars) population. We note that the two youngest stars belong to Pismis 24 . In addition, if several young stars in NGC 6357 trace Pismis 24 several stars of similar age are noted in the external parts of NGC 6357 (Fig. 4). Some of these stars are identified as runaway stars by Gvaramadze et al. (2011) expelled from the stellar cluster Pismis 24 or AH03J1725-34.4. This was already reported by Walborn et al. (2014) who found, at the periphery of the 30 Doradus LMC (Large Magellanic Cloud) nebula, a sample of $\mathrm{O}$ stars in an early evolutionary state, which they assumed were expelled (from the 30 Dor clusters) runaway stars.

Kuhn et al. (2014) identified young stellar clusters in NGC 6357 and NGC 6334. For NGC 6357 Getman et al. (2014) show that the age of the low-mass pre-main-sequence (PMS) stars belonging to different clusters, as well as the more uniformly distributed stars, have similar ages of 1.0 to $1.5 \mathrm{Myr}$ suggesting that the recent star formation proceeded nearly simultaneously across the region. For NGC 6334, they found clusters with ages between 0.7 and $2.3 \mathrm{Myr}$ and a mean age of the dispersed population of 1.9 Myr. Because we found OB stars with ages of $\sim 4.6 \mathrm{Myr}$ in NGC 6357 we suspect that a first star formation event could have shaped the central cavity (and then the larger scale filaments), while the main star formation burst occurred $\sim 1.4$ Myr ago. For NGC 6334, a star formation burst occurred 4 Myr ago forming the optically visible H II regions and has subsequently continued to form the youngest clusters listed by Getman et al. (2014).

In parallel, we can estimate the "immediate past" SFE and SFR. If we assume that our O-type star census is complete, we can estimate the total stellar mass expected to have been formed in both regions. Adopting the Salpeter initial mass function $\left(\mathrm{d} N / \mathrm{d} M=\mathrm{A} \mathrm{m}^{-2.35}\right)$ and a minimum cut-off mass of $0.08 M_{\odot}$ we estimate (following Nguyen et al. 2015) a total stellar mass of $4491 \pm 898 M_{\odot}$ (upper stellar mass $41.6 M_{\odot}$ ) and $8528 \pm 1968 M_{\odot}$ (upper stellar mass $77.8 M_{\odot}$ ) for NGC 6334 and NGC 6357, respectively. For NGC 6357 this is in agreement 
with the stellar mass of $2-6 \times 10^{3} M_{\odot}$ found for Pismis 24 by Massi et al. (2015). Adopting as total gas mass $M_{\text {tot }}=$ $2.3 \times 10^{5} M_{\odot} \quad$ (Willis et al. 2013, Schneider et al. 2015) and $4 \times 10^{5} M_{\odot}$ (Cappa et al. 2011) for NGC 6334 and NGC 6357, respectively, we determine SFEs of $0.019_{-0.007}^{+0.008}$ and $0.021_{-0.003}^{+0.004}$. These SFEs are in the range 0.002-0.2 found for Galactic molecular clouds (Murray 2011; Franco et al. 1994), in agreement with the inner Galaxy molecular cloud median value of 0.02 (Myers et al. 1986) and consistent with typical SFEs estimated for moderate star formation activity star-forming regions (Federrath \& Klessen 2013).

To evaluate the "immediate past" SFR we assume that the star-forming timescale is the age of the oldest massive star used for the SFE calculation (4 and 5 Myr for NGC 6334 and NGC 6357 respectively). We obtain a $S_{F R}$ imm.past equal to $1.1 \times$ $10^{3} \pm 300 M_{\odot} \mathrm{Myr}^{-1}$ and $1.7 \times 10^{3} \pm 400 M_{\odot} \mathrm{Myr}^{-1}$ for NGC 6334 and NGC 6357, respectively, which translate into $\log \left(\Sigma_{\mathrm{SFR}}\right)=$ 0.07 (in $933 \mathrm{pc}^{2}$ ) and $0.13\left(1260 \mathrm{pc}^{2}\right) M_{\odot} \mathrm{yr}^{-1} \mathrm{kpc}^{-2}$, respectively. This places both regions into the mini-starburst regime of the Schmidt-Kennicutt relation (following Fig. 7 of Nguyen et al. 2015). For NGC 6334, these values can be compared with the "recent" SFR evaluated from young stellar objects (YSOs). Indeed, for NGC 6334, Willis et al. (2013) give a total mass for class II and I YSOs of $2000 M_{\odot}$ (in $600 \mathrm{pc}^{2}$ ). Assuming a mean lifetime of $2 \mathrm{Myr}$ for class II YSOs (Evans et al. 2009) this gives $S F R_{\text {recent }}=1 \times 10^{3} M_{\odot} \mathrm{Myr}^{-1}$, $\log \left(\Sigma_{\mathrm{SFR}}(\right.$ recent $\left.)\right)=0.22$. From Tigé et al. (2017), we can also evaluate for NGC 6334 the "present" SFR. Tigé et al. (2017), based on Herschel observations, give a census of 32 high-mass protostars (in a studied area of $1119 \mathrm{pc}^{2}$ ) and a statistical lifetime of $3 \times 10^{5} \mathrm{yr}$. Assuming that these protostars have masses between 8 and $50 M_{\odot}$ we estimate $S F R_{\text {present }}=1.6 \times 10^{4} M_{\odot} \mathrm{Myr}^{-1}$ and $\log \left(\Sigma_{\mathrm{SFR}}\right.$ (present) $)=1.15$. We can then imagine that NGC 6334 is presently experiencing a star formation burst.

\subsection{X-ray counterpart}

The impact of X-ray emission on molecular clouds can regulate star formation and associated timescales. In recent simulations Hocuk \& Spaans (2010) note that star formation can be induced and delayed by X-rays and that massive stars can form with high efficiency. If the X-ray impact of YSOs is negligible it could be more important when massive stars are present (e.g. Feigelson 1997). X-ray emission from massive stars can be generated by different kinds of stellar wind shocks (e.g. Rauw et al. 2015; Chlebowski et al. 1989; Ezoe et al. 2006). Hence, X-ray emission can also be used to identify massive stars with strong winds (Povich et al. 2013).

To investigate this we cross-correlate (cone search $2^{\prime \prime}$ ) our O-B3 stellar sample with the MOXC sample of Chandra/ACIS $\mathrm{X}$-ray point sources from Townsley et al. (2014) to identify $\mathrm{X}$-ray emitting stars. The MOXC catalogue covers the main ridge of NGC 6334 (the regions GUM 61 and H II 351.2+0.5 have not been covered) and most of NGC 6357 (except for region G353.43+0.46) corresponding to about an area of $0.16^{\circ 2}$ and $0.44^{\circ 2}$ for NGC 6334 and NGC 6357, respectively. We find 29 O-B3 stars with an X-Ray counterpart (16 from the present sample and 9 added from the literature). From the catalogued MOXC X-Ray total energy flux we determine the extinction corrected X-ray flux (following Wilms et al. 2000) and the X-Ray luminosity $\left(L_{\mathrm{X}}\right)$ using the previously determined stellar distances and extinction (Table 2). We then investigated the relation between X-Ray and bolometric extinction corrected fluxes (Fig. 5)

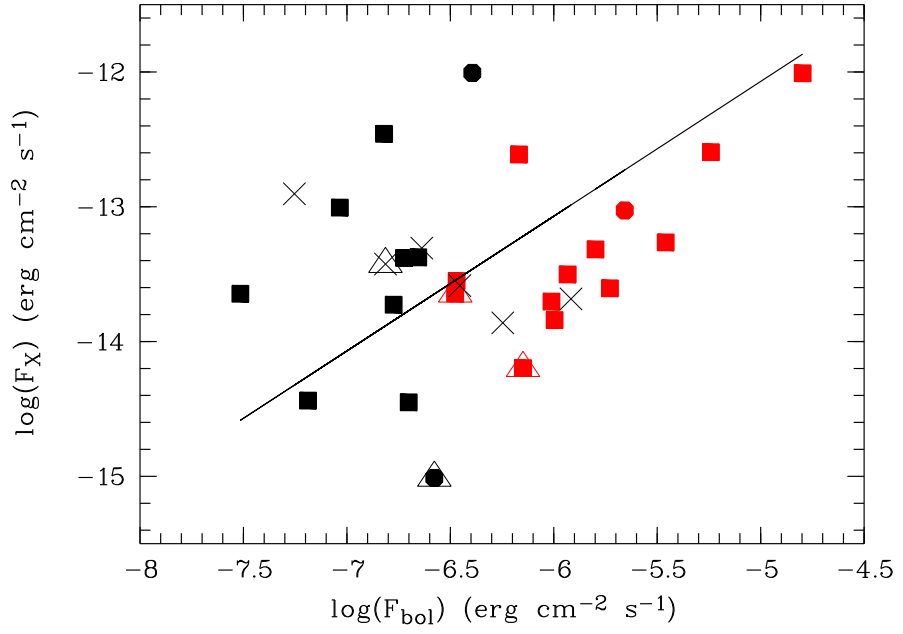

Fig. 5. Relation between X-ray and bolometric luminosity. The stars belonging to NGC 6357 and NGC 6334 are indicated by squares and circles, respectively. The $\mathrm{O}$ and $\mathrm{B}$ stars are coded as red and black symbols. Symbols marked by a triangle are stars with poor spectra, while crosses indicate stars with distances greater than $3 \mathrm{kpc}$ or less than $1.1 \mathrm{kpc}$. The $\mathrm{X}$-ray fluxes are evaluated in the [0.5-8 keV] energy band and corrected for interstellar extinction. The line corresponds to the scaling relation $\log \left(L_{\mathrm{X}} / L_{\mathrm{bol}}\right)=-7.07$.

and obtain the following scaling law ${ }^{4}$ :

$\log \left(L_{\mathrm{X}} / L_{\mathrm{bol}}\right)=-7.07 \pm 0.76$.

This result is in agreement with the well-established relation of Chlebowski et al. (1989) and that found for other regions, as given by Nazé et al. (2011) for O stars in the Carina complex and Rauw et al. (2015) for OB stars in the CygOB2 association. Previously, Wang et al. (2007) led an X-ray census of young stars in NGC 6357. They found a similar scaling relation. In addition, we confirm spectroscopically the B-type status for four OB stars candidates which we have in common (stars 140, 120, 225, and 527 listed in their Table 7). We also confirm spectroscopically eight of the OB candidates listed by Povich et al. (2017): their stars 5 and 8 of NGC 6334 and 2, 3, 17, 18, 19, and 22 of NGC 6357.

Just as reported by Antokhin et al. (2008) we also find an $\mathrm{X}$-ray power demarcation between $\mathrm{B}$ and $\mathrm{O}$ stars corresponding to $L_{\mathrm{bol}} \sim 1.4 \times 10^{38} \mathrm{erg} / \mathrm{s}$.

Amid our 29 X-ray OB star sub-sample, $24\left(\sim 54\right.$ stars/ $\left.{ }^{\circ 2}\right)$ and $5\left(\sim 31{\left.\text { stars } /{ }^{2}\right)}^{2}\right.$ are located toward NGC 6357 and NGC 6334, respectively. Despite the high level of incompleteness of our sample, we can thus expect that molecular clouds in NGC 6357 are likely to be more affected by X-rays and wind than in NGC 6334. The spectral type analysis shows us that 21 stars are main-sequence stars, while 6 are giants, and that 16 and 13 are B- and O-type stars, respectively. In NGC 6357, 12 of the X-ray stars are in the central cavity, mainly belonging to Pismis 24, 6 are located at the border of the $\mathrm{H}$ II region G353.2+0.7, within class I and class II YSOs aggregates as listed by Fang et al. (2012), also identified as cluster "F" by Kuhn et al. (2014), while 2 are isolated in the external region of NGC 6357. These two stars are associated with compact WISE$22 \mu \mathrm{m}$ emission, one of them certainly powering the $\mathrm{H}$ II region $\mathrm{G} 352.838+0.934$. The five X-ray OB stars observed in the direction of NGC 6334 are located in the direction of GUM 64b and

\footnotetext{
4 The scaling factor is obtained by averaging the $\log \left(L_{\mathrm{X}} / L_{\mathrm{bol}}\right)$ other the
} sample. The line plotted in Fig. 5 is then $\log \left(L_{\mathrm{X}}\right)=\log \left(L_{\mathrm{bol}}\right)-7.07$. 
D. Russeil et al.: NGC 6334 and NGC 6357

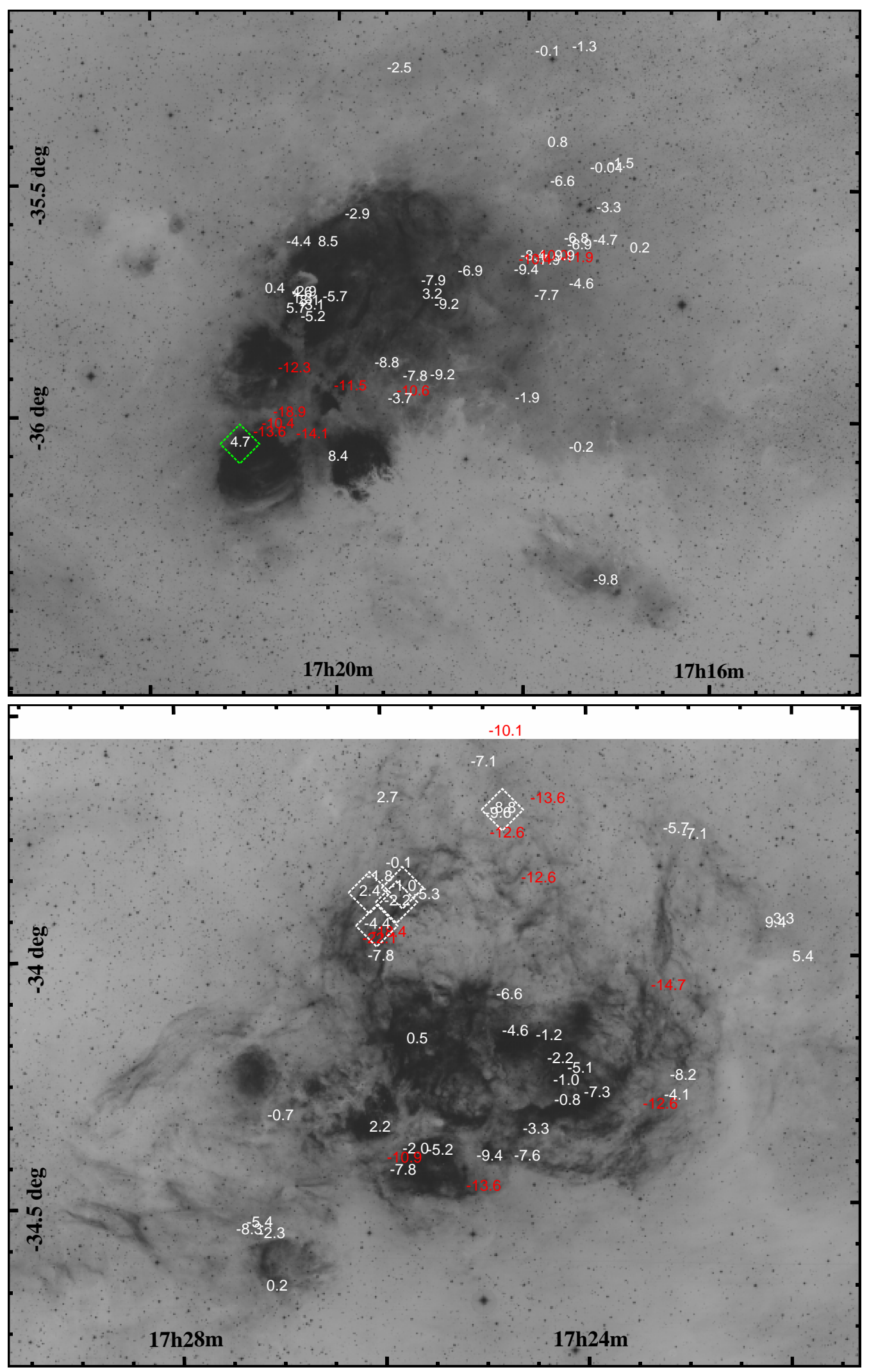

Fig. 6. Velocity (Vlsr in $\mathrm{km} \mathrm{s}^{-1}$ ) measurements overlaid on the $\mathrm{H} \alpha$ images of NGC 6334 (upper panel) and NGC 6357 (lower panel). Velocities more negative than $-10 \mathrm{~km} \mathrm{~s}^{-1}$ are indicated in red. The diamond symbols are discussed in the text.

GUM 64c, two evolved H II regions located on either side of the molecular ridge (Tigé et al. 2017). They are found in small YSO groups of class I and II listed by Willis et al. (2013) and also identified as clusters " $K$ " and "G" by Kuhn et al. (2014).

\subsection{Nebular information}

For the $1042 \mathrm{dF}$ fibres placed on nebular components across the two main regions studied, the resultant spectra are, 


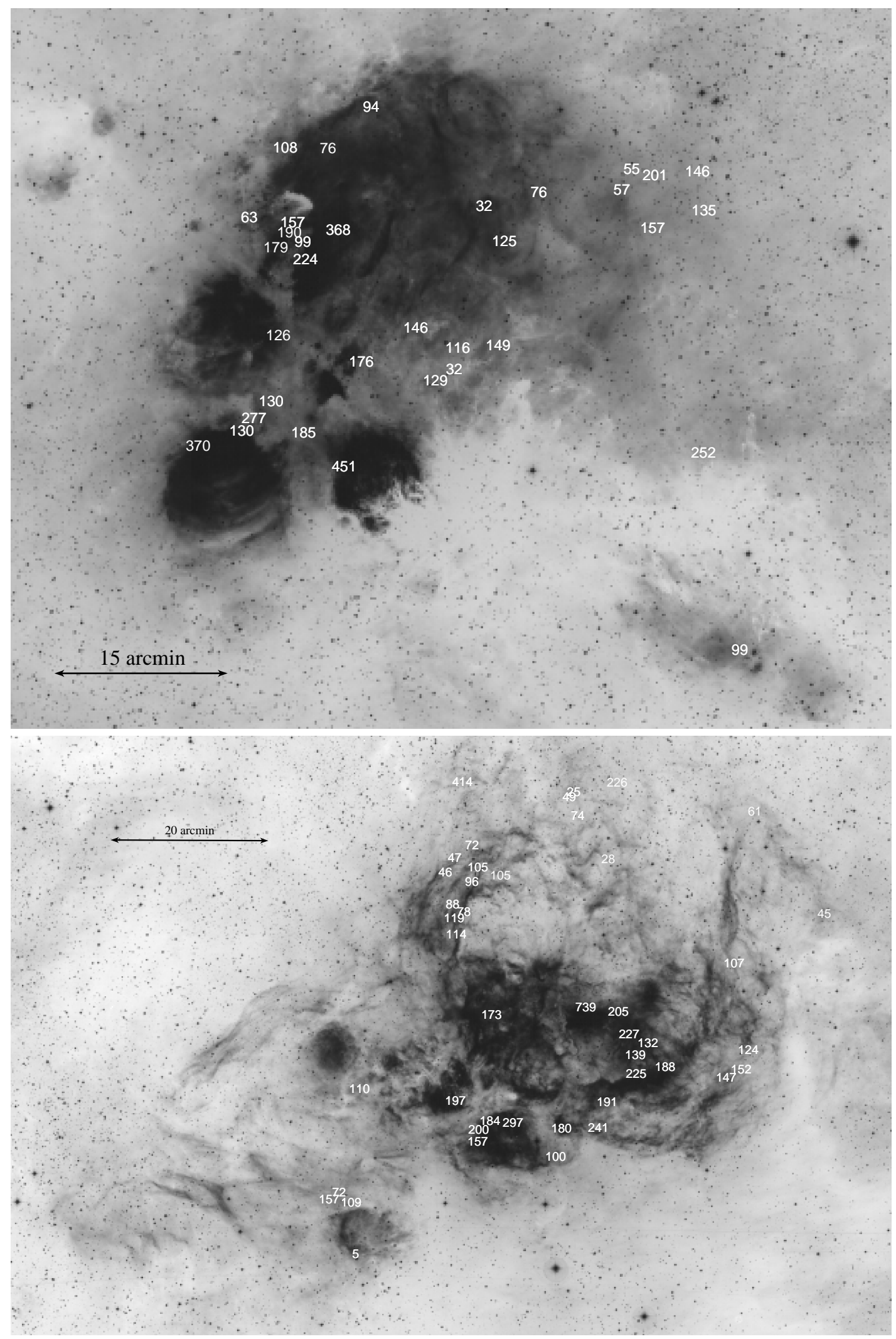

Fig. 7. Spatial locations of individual electron density measurements obtained from nebula spectra (in $\mathrm{cm}^{-3}$ ) overlaid on the $\mathrm{H} \alpha$ images of NGC 6334 (upper panel) and NGC 6357 (lower panel).

unsurprisingly, completely dominated by nebular emission lines that allow us to probe the ionised gas properties at different positions over both NGC 6334 and NGC 6357 (Figs. 6 and 7). We focus on the red part of the spectrum which is less affected by extinction and use the well-known nebular lines of $\mathrm{H} \alpha$, [NII], and $[\mathrm{SII}]$ to derive physical conditions of the ionised gas including electron density. In addition, despite the low resolution $\left(R \sim 1200\right.$ at $\mathrm{H} \alpha$ ) giving a $\sim 24 \mathrm{~km} \mathrm{~s}^{-1}$ uncertainty based on the 


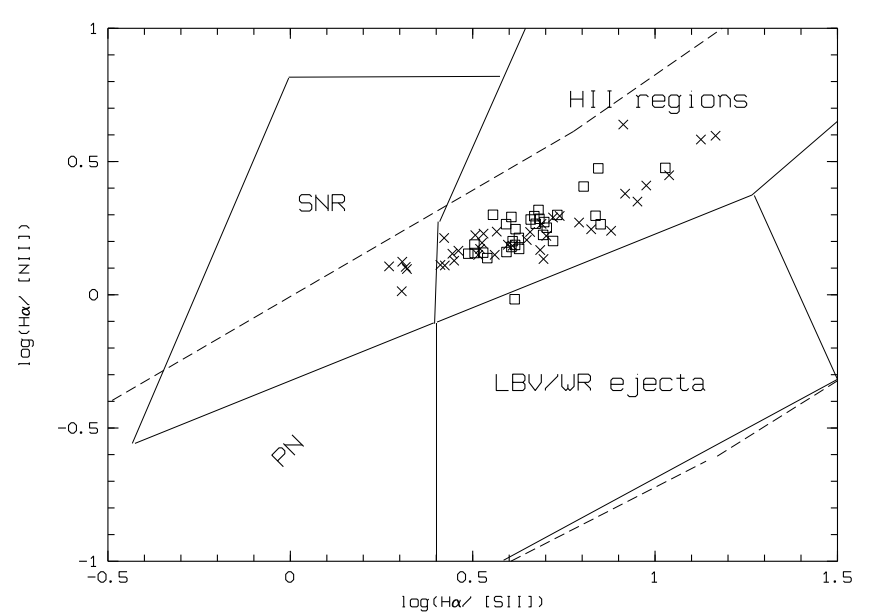

Fig. 8. Line ratios from nebular spectra. Squares and crosses are for positions towards NGC 6334 and NGC 6357 respectively. The delimitation boxes are from Frew \& Parker (2010) and Sabin et al. (2013).

Gaussian fits, we can also derive the Vlsr velocity from the observed emission lines, particularly the $\mathrm{H} \alpha$ line which is usually the strongest.

Velocities: the $\mathrm{H} \alpha$ velocities we obtain (Fig. 6-upper panel) are in overall agreement with the Fabry-Perot interferometer $\mathrm{H} \alpha$ velocities presented in Russeil et al. (2016). In particular in NGC 6357 the $-20 \mathrm{~km} \mathrm{~s}^{-1}$ arc-like feature at $\alpha, \delta=$ $17 \mathrm{~h} 22 \mathrm{~m} 59.5 \mathrm{~s},-33^{\circ} 57^{\prime} 14^{\prime \prime}$ (north-east) located in a $\sim-3 \mathrm{~km} \mathrm{~s}^{-1}$ filamentary structure is well recovered. The ionised gas in the open side of the large-scale NGC 6357 cavity has a velocity between -7 and $-14 \mathrm{~km} \mathrm{~s}^{-1}$, while the inner cavity surrounding Pismis 24 shows a -1 to $-7 \mathrm{~km} \mathrm{~s}^{-1}$ velocity range. The velocity of GM 1-24 $\left(-10 \mathrm{~km} \mathrm{~s}^{-1}\right)$ is also recovered. Negative velocity up to $\sim-19 \mathrm{~km} \mathrm{~s}^{-1}$ can be noted at locations between the H II regions GUM 61, GUM 64b, and H II $351.2+0.5$. They suggest that this area can be quite turbulent and could underline possible kinematic mixing/interaction of the $\mathrm{H}$ II regions.

Physical conditions from the sampled nebula positions across NGC 6334 and NGC 6357: in this part we use only spectra with $\mathrm{S} / \mathrm{N}$ higher than 20 for all the red nebular lines. The measured electron densities were derived in standard fashion from the observed [SII] $\lambda \lambda$ 6716/6731 ratios in each spectrum (Osterbrock \& Ferland 2006). The lack of flux calibration is not an issue as the lines are a doublet and are adjacent. By selecting spectra with $\mathrm{S} / \mathrm{N}$ higher than 20 for both [SII] lines more reliable results are obtained. Electron temperatures of $7000 \mathrm{~K}$ and $6000 \mathrm{~K}$ for NGC 6334 (Copetti et al. 2000) and NGC 6357 (Quireza et al. 2006) were used in combination with the line ratio to derive the electron density $n_{\mathrm{e}}$ (Fig. 7) using the FIVEL code (De Robertis et al. 1987). Derived electron densities varied from 32 to $370 \mathrm{~cm}^{-3}$ in NGC 6334 and from 28 to $739 \mathrm{~cm}^{-3}$ in NGC 6357. The highest electron density value is found towards G353.2+0.9, the ionised gas region facing Pismis 24, in broad agreement with Lortet et al. (1984) and Bohigas et al. (2004) who found density up to $\sim 3000 \mathrm{~cm}^{-3}$ for this region. In NGC 6357 we find higher density $\left(n_{\mathrm{e}} \sim 100 \mathrm{~cm}^{-3}\right)$ towards the larger scale filamentary structures than Lortet et al. (1984) who note $n_{\mathrm{e}}$ between 40 and $50 \mathrm{~cm}^{-3}$ for the filamentary structures near G353.2+0.9. In NGC 6334, Copetti et al. (2000) show density variation between $\sim 200 \mathrm{~cm}^{-3}$ and $500 \mathrm{~cm}^{-3}$ through H II $351.2+0.5$ (GUM 62) and GUM 64a (G351.17+0.70) in agreement with our global measurements.
The $\log (\mathrm{H} \alpha /[\mathrm{NII}])$ versus $\log (\mathrm{H} \alpha /[\mathrm{SII}])$ emission line ratio diagnostic diagram (Fig. 8) allows us to locate the measured nebula sample points across the regions in relation to the loci of other known emission object types. The most up-to-date diagnostic diagrams of this type are provided by Frew \& Parker (2010), Sabin et al. (2013), and Frew et al. (2014) and are recommended for use. In these plots the typical regions occupied by $\mathrm{H}$ II regions (photoionised gas), PNe, supernova remnants (SNRs) (shock-heated gas), luminous blue variables (LBVs), and Wolf-Rayet shells are reproduced from these papers. In Fig. 8 we note that most of the points fall inside the $\mathrm{H}$ II region box, as expected, while few positions fall in the SNR box suggesting they are shock heated. These last points are all located in the direction of NGC 6357 (white diamonds on Fig. 6) suggesting that a strong event occurred in this region. One position is in the LBV/Wolf-Rayet box. This fibre position (green diamond in Fig. 6) is in the direction of the $\mathrm{H}$ II region $\mathrm{H}$ II $351.2+0.5$ between the CS90 bubble edge and the H II 351.2+0.5 PDR underlining any particular physical condition.

\section{Conclusions}

Based on observations performed with the AAOmega spectrograph we were able to spectroscopically classify a significant number (109) of OB stars in the direction of NGC 6334 and NGC 6357. Using more accurate distances to these OB stars, their star formation history could be better investigated. This work suggests that the star formation in NGC 6334 and NGC 6357 started 4 to $4.5 \mathrm{Myr}$ ago. NGC 6357 seems to have experienced a first star-forming event $\sim 4.5 \mathrm{Myr}$ ago, mainly located in the prominent cavity, that could be at the origin of the shock heated filamentary structures and the runaway stars, and a second more recent event ( $1.5 \mathrm{Myr}$ ago $)$ which explains the young stellar population observed by Getman et al. (2014). For NGC 6334, from massive star formation studies of the HerschelHOBYS project (Tigé et al. 2017), we suspect that it is presently experiencing a star formation burst. The next step is also to establish the star formation history for NGC 6357 with similar Herschel-HOBYS project (Tigé et al. 2017) data. We also expect to refine our distance estimates, to confirm the nature of the possible identified runaway stars, and to clarify possible binarity with data from the incoming ESA-Gaia database.

\section{References}

Anderson, L. D., Bania, T. M., Balser, D. S., et al. 2011, ApJS, 194, 32 Anderson L. D., Bania, T. M., Balser, D. S., et al. 2014, ApJS, 212,1 Antokhin, I. I., Rauw, G., Vreux, J.-M., et al. 2008, A\&A, 477, 593 Arias, J. I., Walborn, N. R., Simòn Díaz, S. , et al. 2016, AJ, 152, 31 Bohigas, J., Tapia, M., Roth, M., et al. 2004, J. Korean Astron. Soc., 37, 281 Bronfman, L., Nyman, L.-A., \& May, J. 1996, A\&AS, 115, 81 Cappa, C. E., Barbá, R., Duronea, N. U., et al. 2011, MNRAS, 415, 2844 Cardelli, J. A., Clayton, G. C., \& Mathis, J. S. 1989, ApJ, 345, 245 Carraro, G. 2011, A\&A, 536, A101

Chlebowski, T., Harnden, F. R., \& Sciotino, S. 1989, ApJS, 341, 427 Chibueze, J. O., Omodaka, T., Handa, T., et al. 2014, ApJ, 784, 114 Churchwell, E., Watson, D. F., \& Povich, M. S. 2007, ApJ, 670, 428 Copetti, M. V. F., Mallmann, J. A. H., Schmidt, A. A., et al. 2000, A\&A, 357, 621

Crowther, P. A., Lennon, D. J., \& Walborn, N. R. 2006, A\&A, 446, 279 Dashs, J., Kaiser, D., Nikolov, A., et al. 1982, A\&AS, 50, 261 De Robertis, M. M., Dufour, R. J., \& Hunt, R. W. 1987, JRASC, 81, 195 Ezoe, Y., Kokubun, M., Makishima, K., et al. 2006, ApJ, 638, 860 Evans, N. J., II, Dunham, M. M., Jorgensen, J. K., et al. 2009, ApJS, 181, 321 Fang, M., van Boekel, R., King, R. R., et al. 2012, A\&A, 539, A119 Federrath, C., \& Klessen, R. 2013, ApJ, 763, 51

Feigelson, E. D. 1997, Mem. Soc. Astron. Ital., 68, 1007 Franco, J., Shore, S. N., \& Tenorio-Tagle, G. 1994, ApJ, 436, 795 
Frew, D. J., \& Parker, Q. A. 2010, PASA, 27, 129

Frew, D. J., Bojicic, I. S., Parker, Q. A., et al. 2014, MNRAS, 440, 1345

Getman, K., Feigelson, E., Kuhn, M., et al. 2014, ApJ, 787, 108

Gvaramadze, V. V., Kniazev, A. Y., Kroupa P., et al. 2011, A\&A, 535, A29

Hauck, B., \& Mermilliod, M. 1998, A\&AS, 129, 431

Hocuk, S., \& Spaans, M. 2010, A\&A, 522, A24

Hou, L. G., \& Han, J. L. 2014, A\&A, 569, A125

Humphreys, R. M., \& McElroy, D. B. 1984, ApJ, 284, 565

Kharchenko, N. V., Piskunov, A. E., Roeser, S., et al. 2005, A\&A, 438, 1163

Kharchenko, N. V., Piskunov, A. E., Schilbach, E., et al. 2013, A\&A, 558, A53

Kharchenko, N. V., Piskunov, A. E., Schilbach, E., et al. 2016, A\&A, 585, A101

Kuhn, M., Feigelson, E., Getman, K., et al. 2014, ApJ, 787, 107

Lejeune, T., \& Schaerer, D. 2001, A\&A, 366, 538

Lima, E.F., Bisa, E., Bonatto, C., et al. 2014, A\&A, 568, A16

Lindegren, L., Lammers, U., Bastian, U., et al. 2016, A\&A, 595, A4

Lortet, M. C., Testor, G., \& Niemela, V. 1984, A\&A, 140, 24

Mackey, J., Haworth, T. J., Gvaramadze, V. V., et al. 2016, A\&A, 586, A114

Maíz Apellániz, J., Walborn, N. R., Morrell, N. I., et al. 2007, ApJ, 660, 1480

Maíz Apellániz, J., Sota, A., Arias, J. I., et al. 2016, ApJS, 224, 4

Martins, F., \& Plez, B. 2006, A\&A, 457, 637

Martins, F., Schaerer, D., \& Hillier, D. J. 2005, A\&A, 436, 1049

Massey, P., DeGioia-Eastwood, K., \& Waterhouse, E. 2001, AJ, 121, 1050

Massi, F., Giannetti, A., Di Carlo, E., et al. 2015, A\&A, 573, A95

Mel'Nik, A. M., Sitnik, T. G., Dambis, A. K., et al. 1998, AstL, 24, 594

Morales, E. F. E., Wyrowski, F., Schuller, F., et al. 2013, A\&A, 560, A76

Muñoz, D. J., Mardones, D., Garay, G., et al. 2007, ApJ, 668, 906

Murray, N. 2011, ApJ, 729, 133

Myers, P. C., Dame, T. M., Thaddeus, P., et al. 1986, ApJ, 301, 398

Nazé, Y., Broos, P. S., Oskinova, L., et al. 2011, ApJS, 194, 7

Neckel, T. 1978, A\&A, 69, 51

Neckel, T. 1984, A\&A, 137, 58

Nguyen, H., Nguyen-Luong, Q., Martin, P. G., et al. 2015, ApJ, 812, 7

Osterbrock, D. E., \& Ferland, G. J. 2006, Astrophysics Of Gaseous Nebulae And

Active Galactic Nuclei, 2nd edn. (University Science Books)

Panagia, N. 1973, AJ, 78, 929

Parker, Q. A., Philipps, S. Pierce, M., et al. 2005, MNRAS, 362, 689
Persi, P., \& Tapia, M. 2008, in Handbook of Star Forming Regions, Vol. II, Ed. B. Reipurth, 456

Pinheiro, M., Copetti, M., \& Oliviera, V. 2010, A\&A, 521, A26

Povich, M. S., Kuhn, M. A., Getman, K. V., et al. 2013, ApJS, 209, 31

Povich, M. S., Busk, H. A., Feigelson, E. D., et al. 2017, ApJ, 838, 61

Quireza, C., Rood, R. T., Bania, T. M., et al. 2006, ApJ, 653, 1226

Rauw, G., Nazé, Y. Wirght, N. J., et al. 2015, ApJS, 221, 1

Roslund, C. 1966, Arkiv. Astron., 4, 73

Russeil, D., Adami, C., \& Georgelin, Y. M. 2007, A\&A, 470, 161

Russeil, D., Zavagno, A., Motte, F., et al. 2010, A\&A, 515, A55

Russeil, D., Zavagno, A., Adami, C., et al. 2012, A\&A, 538, A142 (Paper I)

Russeil, D., Tigé, J., Adami, C., et al. 2015, A\&A, 587, A135

Sabin, L., Parker, Q. A., Contreras, M. E., et al. 2013, MNRAS, 431, 279

Schmidt-Kaler, T. 1983, Landolt-Bornstein, New series group VI, Springer, 23, 14

Schneider, N., Bontemps, S., Girichidis, P., et al. 2015, MNRAS, 453, L41

Sharp, R., Saunders, W., Smith, G., et al. 2006, Proc. SPIE, 6269

Simpson, R. J., Povich, M. S., Kendrew, S., et al. 2012, MNRAS, 424, 2442

Sota, A., Maíz Apellániz, J., Walborn, N. R., et al. 2011, ApJS, 193, 24

Sota, A., Maíz Apellániz, J., Morrell, N. I., et al. 2014, ApJS, 211, 10

Sung, H., Lim, B., Bessel, M. S., et al. 2013, J. Korean Astron. Soc., 46, 103

Tigé, J., Motte F., Russeil, D., et al. 2017, A\&A, 602, A77

Townsley, L. K., Broos, P. S., Garmire, G. P., et al. 2014, ApJS, 213, 1

Trundle, C., Dufton, P. L., Hunter, I., et al. 2007, A\&A, 471, 625

Vacca, W. D., Garmany, C. D., \& Shull, J. M. 1996, ApJ, 460, 914

Walborn, N. R. 1980, ApJS, 44, 535

Walborn, N. R. 1982, AJ, 87, 1300

Walborn, N. R., \& Fitzpatrick, E. L. 1990, PASP, 102, 379

Walborn, N. R., Sana, H., Símon-Díaz, S., et al. 2014, A\&A, 564, A40

Wang, J., Townsley, L. K., Feigelson, E. D., et al. 2007, ApJS, 168, 100

Wenger, T. V., Bania, T. M., Balser, D. S., et al. 2013, ApJ, 764, 34

Willis, S., Marengo, M., Allen, L., et al. 2013, ApJ, 778, 96

Wilms, J., Allen, A., \& McCray, R. 2000, ApJ, 542, 914

Wright, N. J., Drew, J. E., \& Mohr-Smith, M. 2015, MNRAS, 449, 741

Wu, Y. W., Sato, M., Reid, M. J., et al. 2014, A\&A, 566, A17

Zacharias, N., Monet, D. G., Levine, S. E., et al. 2004, A\&AS, 205, 4815 


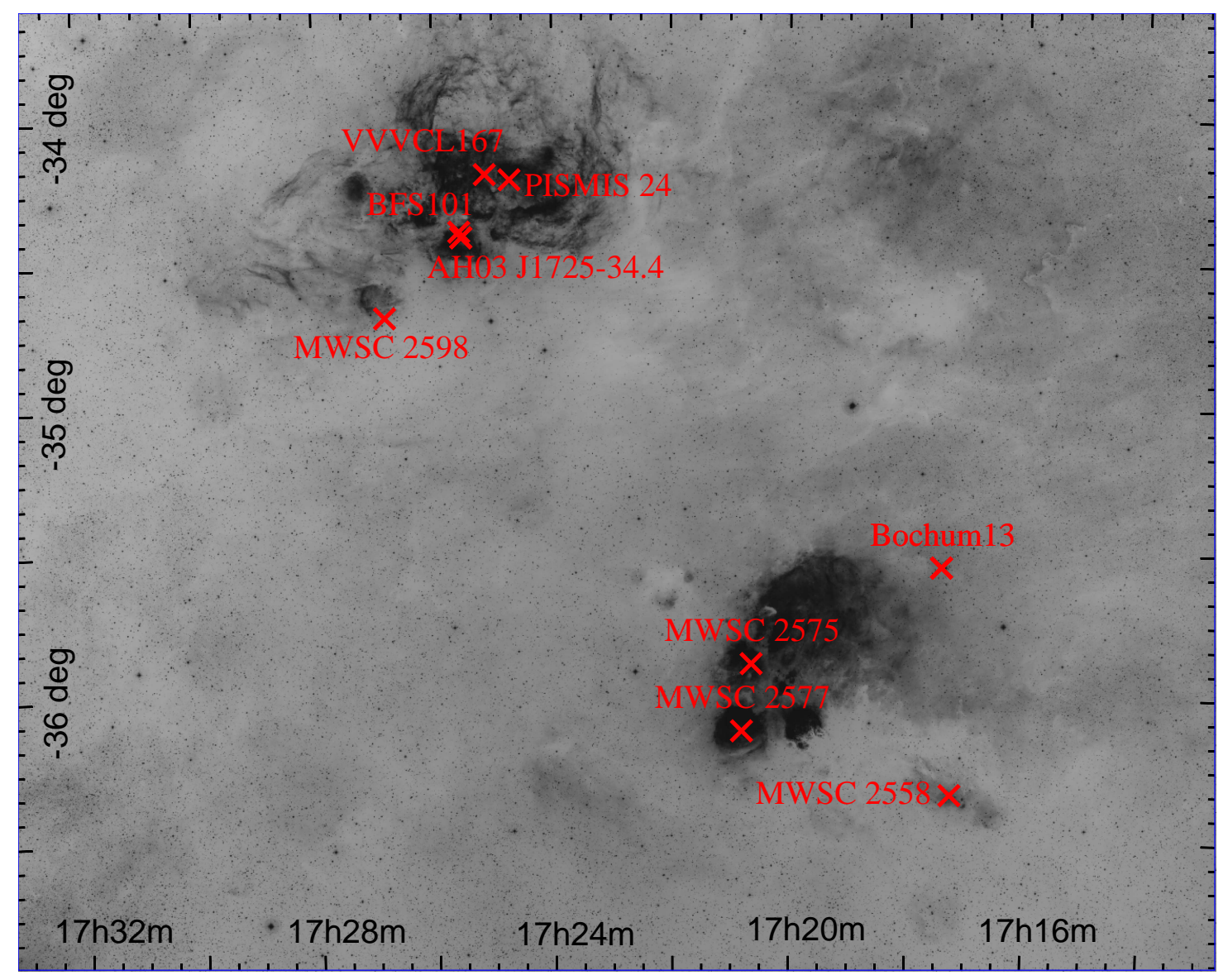

Fig. A.1. Location of the clusters discussed in the text are overlaid on the $\mathrm{H} \alpha$ image.

\section{Appendix A: Additional figures and tables}

Figure A.1 shows the cluster distribution.

Table A. 1 summarises the cross-correlation ( $3^{\prime \prime}$ cone search) of our star sample with the Gaia-DR1 and 2MASS catalogues. In Table A.1 are also indicated the alternative name from the SIMBAD $^{5}$ database and the region towards which the star is observed. The 2MASS "prox" flag is the distance between the source and its nearest neighbour in the point source catalogue.
It can be estimated that if prox $<2^{\prime \prime}$, both stars could contribute to the observed spectra and could bias the classification. If the Gaia flag is 1, this may indicate observational, cross-matching, or processing problems or may indicate stellar multiplicity; in all cases there are probable astrometric or photometric problems. In addition, Fig. A.2 allows us to identify two possible variable/binary stars (stars 30 and 72) as clear outliers. 
Table A.1. Additional stellar information.

\begin{tabular}{|c|c|c|c|c|c|c|c|}
\hline Star no. & Gaia source Ident. & $\begin{array}{l}\text { Gaia } \\
\text { duplicate flag }\end{array}$ & $\begin{array}{l}\text { Gaia } \\
\text { phot_g_mean_mag } \\
\text { mag }\end{array}$ & 2MASS source ident. & $\begin{array}{l}\text { 2MASS } \\
\text { prox. flag } \\
\text { arcsec }\end{array}$ & Region & Other name \\
\hline 1 & 5973950138917230592 & 0 & 14.229113145 & $17160382-3618517$ & 9.5 & GM1-24 & \\
\hline 2 & 5973950345075694976 & 0 & 14.510178835 & $17160431-3616548$ & 7.6 & GM1-24 & \\
\hline 3 & 5973946565504399872 & 0 & 15.033785498 & $17162955-3620558$ & 4.3 & GM1-24 & \\
\hline 4 & 5973933646244126080 & 0 & 14.296968825 & $17163510-3627367$ & 8.2 & GM1-24 & \\
\hline 5 & 5973936154503703424 & 0 & 14.71264494 & $17165359-3619225$ & 14.0 & GM1-24 & \\
\hline 6 & 5973948730168050560 & 0 & 12.808599462 & $17170051-3613362$ & 6.8 & GM1-24 & \\
\hline 7 & 5973935123711518336 & 0 & 14.644419498 & $17170147-3622014$ & 9.5 & GM1-24 & \\
\hline 8 & 5973936017064737024 & 0 & 14.749675417 & $17170247-3620049$ & 7.0 & GM1-24 & \\
\hline 9 & 5973935673467361408 & 0 & 15.104264978 & $17171143-3619393$ & 4.4 & GM1-24 & \\
\hline 10 & 5973935295517186816 & 0 & 14.065299802 & 17171858-3621384 & 8.8 & GM1-24 & \\
\hline 11 & 5975437198328133888 & 0 & 12.060555395 & $17172630-3614476$ & 6.6 & GM1-24 & \\
\hline 12 & 5975438396625217280 & 0 & 14.655115195 & $17172627-3613098$ & 4.2 & GM1-24 & \\
\hline 13 & 5975438843301844992 & 0 & 14.070477442 & $17172771-3609472$ & 3.6 & GM1-24 & \\
\hline 14 & 5975460799175018112 & 0 & 14.217143222 & $17174738-3553467$ & 5.0 & NGC 6334 & \\
\hline 15 & 5975445131134109568 & 0 & 15.175357094 & $17185457-3601043$ & 8.2 & NGC 6334 & \\
\hline 16 & 5975488085104930816 & 0 & 14.497982459 & 17185867-3541489 & 4.1 & NGC 6334 & VdBH 85a C \\
\hline 17 & 5975487840287579136 & 0 & 13.424815398 & $17190547-3542123$ & 6.3 & NGC 6334 & \\
\hline
\end{tabular}

Notes. Full table is available at the CDS.

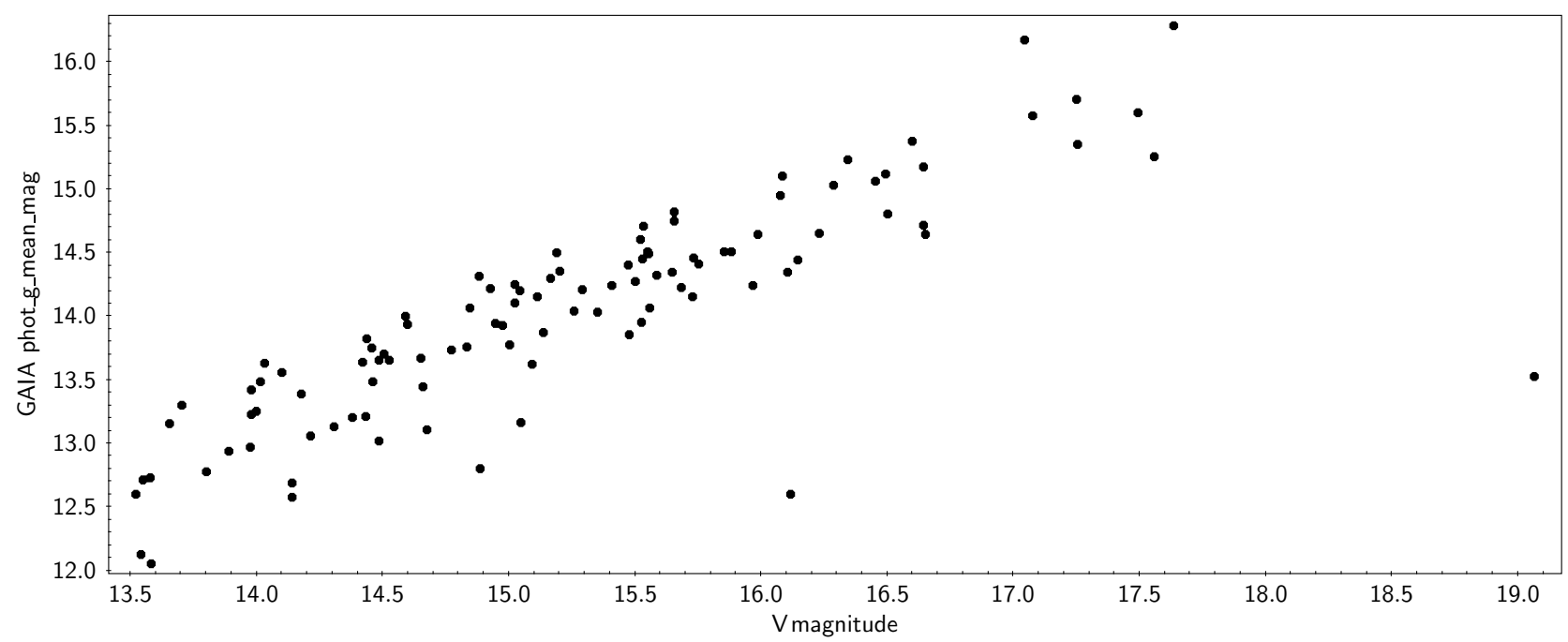

Fig. A.2. Gaia $G$-band photometry versus the $V$-band (from Paper I) photometry. 
Table A.2. Stellar data collected from the literature.

\begin{tabular}{|c|c|c|c|c|c|c|c|c|}
\hline Name & $\begin{array}{l}\text { Equatorial coordinates } \\
\left.\text { J2000 (hms }{ }^{\circ}, \prime \prime\right)\end{array}$ & $\begin{array}{l}V \\
\text { mag }\end{array}$ & $\begin{array}{l}B-V \\
\text { mag }\end{array}$ & Ref. & Spectral type & Ref. & $\begin{array}{l}d \\
\mathrm{kpc}\end{array}$ & $\begin{array}{l}A_{V} \\
\text { mag }\end{array}$ \\
\hline \multicolumn{9}{|c|}{ NGC 6357} \\
\hline HDE319718 = Pis24-1 & $172443.41-341156.5$ & 10.43 & 1.45 & 1 & O3 If* & 1 & $1.39 \pm 0.14$ & $6.10 \pm 0.21$ \\
\hline Pis24-17 & $172444.70-341202$ & 11.84 & 1.49 & 1 & O3 III(f*) & 1 & $2.27 \pm 0.74$ & $6.24 \pm 0.21$ \\
\hline Pis24-2 & $172443.20-341243.5$ & 11.95 & 1.41 & 1 & O5.5 V((f)) & 1 & $1.69 \pm 0.21$ & $6.14 \pm 0.20$ \\
\hline Pis $24-15$ & $172428.86-341450.3$ & 12.44 & 1.18 & 9 & $\mathrm{O} 8 \mathrm{~V}$ & 1 & $2.44 \pm 0.74$ & $5.25 \pm 0.18$ \\
\hline Pis $24-13$ & $172445.68-340939.2$ & 12.32 & 1.33 & 9 & O6.5 V((f)) & 1 & $2.07 \pm 0.74$ & $5.82 \pm 0.20$ \\
\hline Pis 24-3 & $172442.21-341321.0$ & 12.77 & 1.44 & 9 & $\mathrm{O} 8 \mathrm{~V}$ & 1 & $1.86 \pm 0.57$ & $6.17 \pm 0.2$ \\
\hline Pis $24-10$ & $172435.94-341359.9$ & 12.96 & 1.50 & 1 & O9 V & 1 & $1.60 \pm 0.37$ & $6.38 \pm 0.21$ \\
\hline Pis 24-16 & $172444.30-341200$ & 13.02 & 1.60 & 1 & $07.5 \mathrm{~V}$ & 1 & $1.76 \pm 0.58$ & $6.77 \pm 0.22$ \\
\hline Pis $24-12$ & $172442.22-341141.1$ & 13.88 & 1.47 & 1 & $\mathrm{~B} 1 \mathrm{~V}$ & 1 & $1.56 \pm 0.67$ & $6.10 \pm 0.20$ \\
\hline Pis $24-18$ & $172443.20-341142$ & 13.97 & 1.48 & 1 & B0.5 V: & 1 & $1.97 \pm 0.58$ & $6.21 \pm 0.21$ \\
\hline Pis24-19 & $172443.50-341141$ & 14.43 & 1.39 & 1 & $\mathrm{~B} 1 \mathrm{~V}$ & 1 & $1.76 \pm 0.58$ & $6.77 \pm 0.22$ \\
\hline TYC 7370-460-1 & $171815.4-340006.1$ & 11.80 & 1.45 & 9 & $06.5 \mathrm{~V}$ & 2 & $1.39 \pm 0.13$ & $6.28 \pm 0.21$ \\
\hline HD 319881 & $172821.67-343230.4$ & 10.14 & 0.96 & 10 & $\mathrm{O} 5 \mathrm{~V}$ & 2 & $1.30 \pm 0.20$ & $4.97 \pm 0.18$ \\
\hline $2 M A S S$ J17271123-3414349 & $172711.23-341434.9$ & 11.77 & 0.52 & 11 & 07.5 & 2 & $5.52 \pm 1.74$ & $2.93 \pm 0.13$ \\
\hline 2MASS J17220342-3414241 & $172203.43-341424.1$ & 13.59 & 1.26 & 11 & O5.5 & 2 & $4.61 \pm 0.59$ & $5.61 \pm 0.19$ \\
\hline \multicolumn{9}{|c|}{ NGC 6334} \\
\hline CD-35 11465 & $171907.5-353746$ & 11.44 & 0.76 & 7 & B2 IV & 8 & $1.56 \pm 0.58$ & $3.55 \pm 0.22$ \\
\hline HD 319701 & $171916.0-355408$ & 10.10 & 1.08 & 7 & $\mathrm{~B} 1 \mathrm{Ib}$ & 8 & $1.88 \pm 0.93$ & $4.55 \pm 0.26$ \\
\hline HD 319699 & $171930.4-354236$ & 9.63 & 0.80 & 7 & $05 \mathrm{~V}((\mathrm{fc}))$ & 3 & $1.69 \pm 0.26$ & $3.91 \pm 0.23$ \\
\hline CD-3511477 & $172005.0-355638$ & 11.11 & 0.90 & 7 & $\mathrm{~B} 0.5 \mathrm{~V}$ & 4 & $1.33 \pm 0.39$ & $4.20 \pm 0.24$ \\
\hline HD 319697 & $172024.8-354232$ & 10.33 & 0.66 & 7 & $\mathrm{~B} 1 \mathrm{~V}$ & 8 & $1.33 \pm 0.39$ & $4.20 \pm 0.24$ \\
\hline CD-35 11482 & $172026.5-354407$ & 10.70 & 0.73 & 7 & $\mathrm{~B} 0.5 \mathrm{Ve}$ & 6 & $1.46 \pm 0.43$ & $3.59 \pm 0.22$ \\
\hline HD $319703 B$ & $171945.0-360547$ & 11.19 & 1.25 & 7 & $06.5 \mathrm{~V}((\mathrm{f})) \mathrm{z}$ & 3 & $1.37 \pm 0.49$ & $5.58 \pm 0.30$ \\
\hline HD 319703A & $171946.2-360552$ & 10.71 & 1.18 & 7 & $\mathrm{O} 7 \mathrm{~V}((\mathrm{f})) \mathrm{z}$ & 3 & $1.71 \pm 0.52$ & $5.19 \pm 0.29$ \\
\hline CD-35 11484 & $172049.8-355221$ & 11.33 & 0.95 & 7 & $\mathrm{~B} 1 \mathrm{~V}$ & 8 & $1.10 \pm 0.48$ & $4.30 \pm 0.25$ \\
\hline HD 319702 & $172050.6-355146$ & 10.16 & 0.93 & 7 & O8 III & 3 & $1.84 \pm 0.40$ & $4.30 \pm 0.25$ \\
\hline HD 156738 & $172052.7-360421$ & 9.36 & 0.90 & 7 & O6.5 III(f) & 5 & $1.57 \pm 0.52$ & $4.20 \pm 0.24$ \\
\hline
\end{tabular}

Notes. The two stars written in italics have a spectral type from Gvaramadze et al. (2011) but no luminosity class. For the distance and $A_{V}$ determination we assume they are main sequence stars.

References. 1) Massey et al. (2001); 2) Gvaramadze et al. (2011); 3) Sota et al. (2014); 4) Pinheiro et al. (2010); 5) Walborn (1982); 6) Roslund (1966); and 7) Neckel (1978) or Neckel (1984); 8) Persi \& Tapia (2008) and Hauck \& Mermilliod (1998); 9) Russeil et al. (2012); 10) Dashs et al. (1982); 11) Zacharias et al. (2004), NOMAD catalogue. 
Appendix B: The spectra database
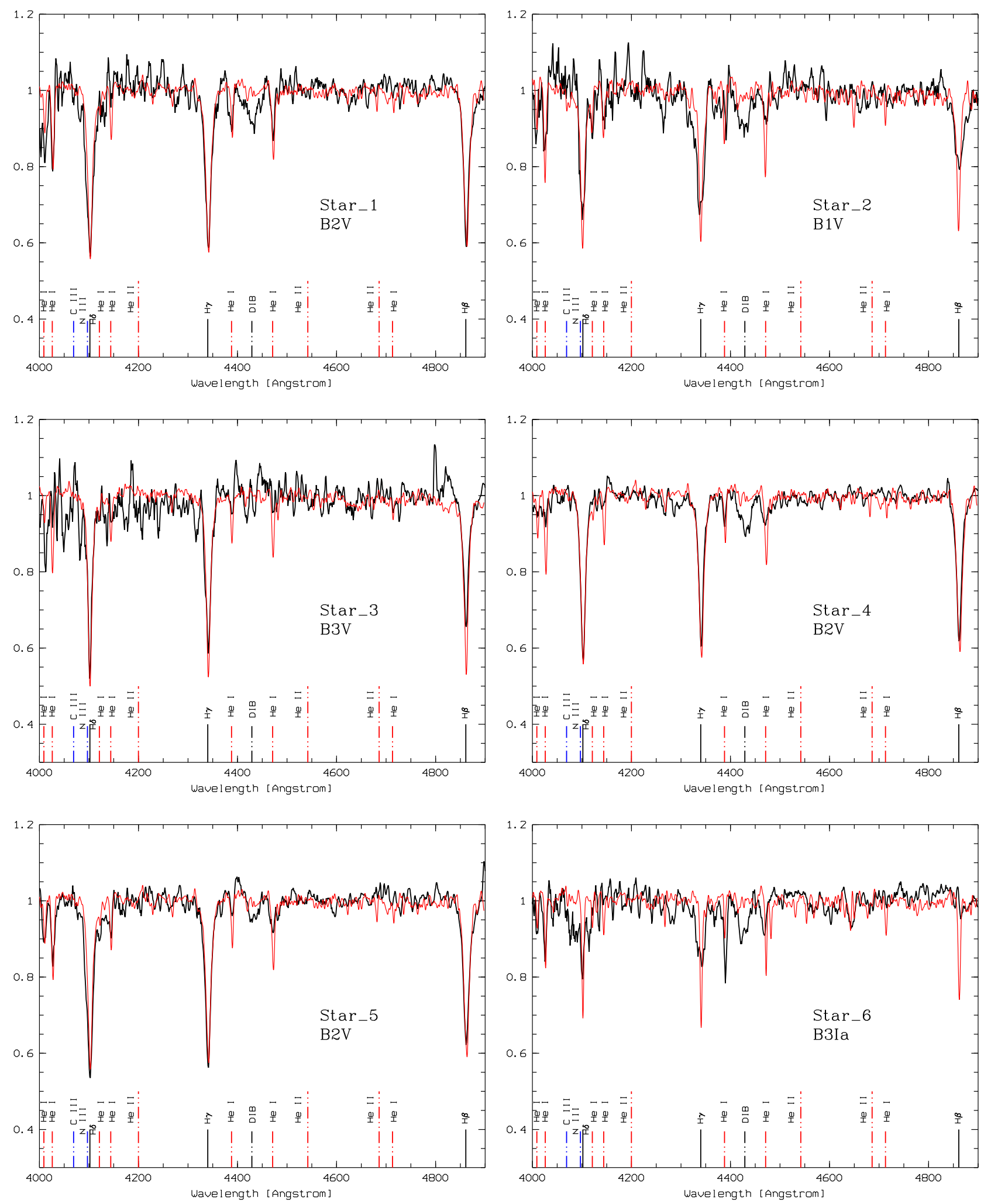

Fig. B.1. Normalised spectra (black curves) of our OB star sample. Tickmarks indicate the expected position of the hydrogen lines (black solid), the diffuse insterstellar absorption band (black dash-dot-dot), the helium I (red dash-dot-dot) and helium II (long red dash-dot-dot) lines, and the CIII-4069 and NIII-4097 lines (blue dash-dot-dot). The red plot is the template corresponding to the indicated spectral type. 
D. Russeil et al.: NGC 6334 and NGC 6357
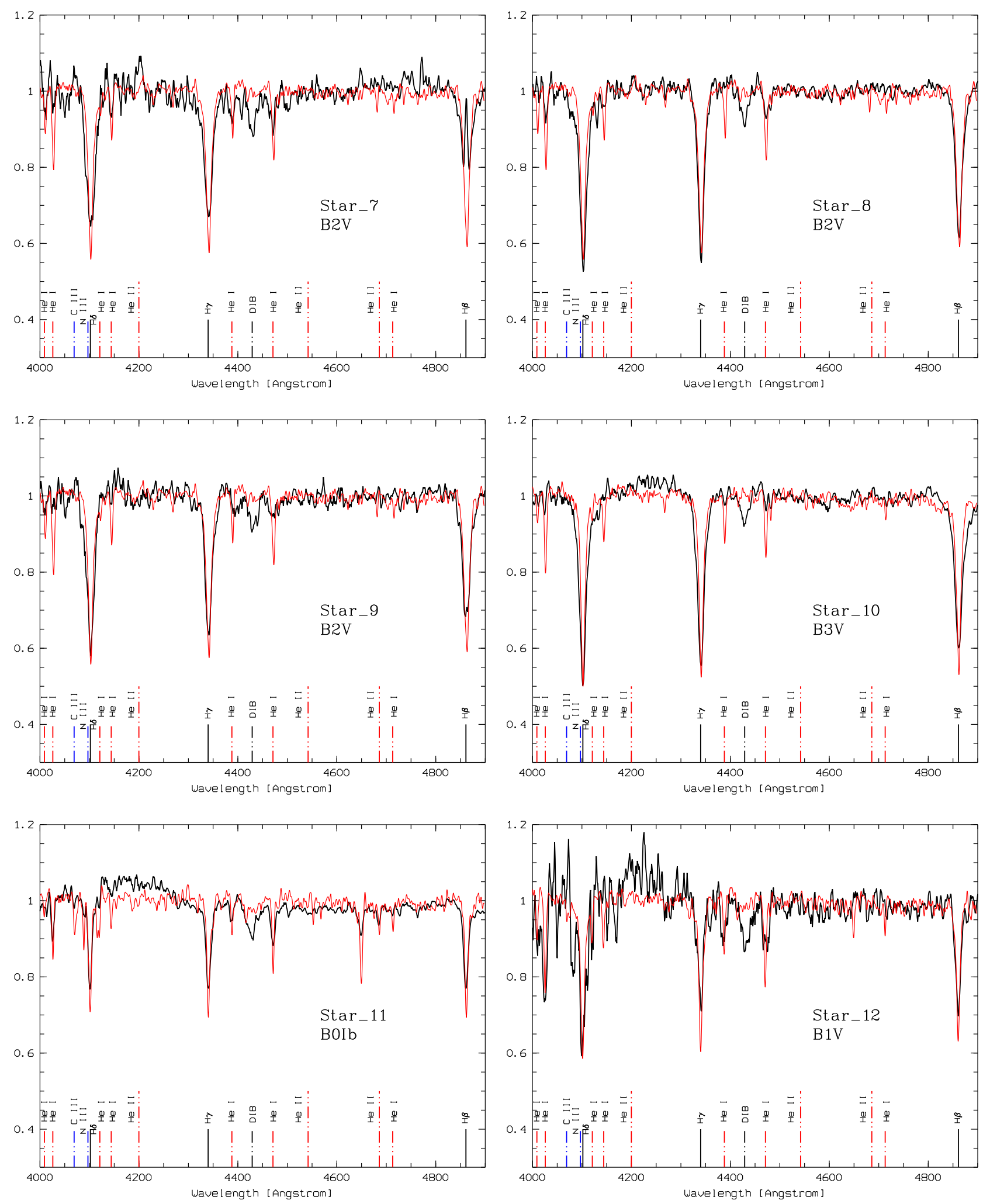

Fig. B.1. continued. 

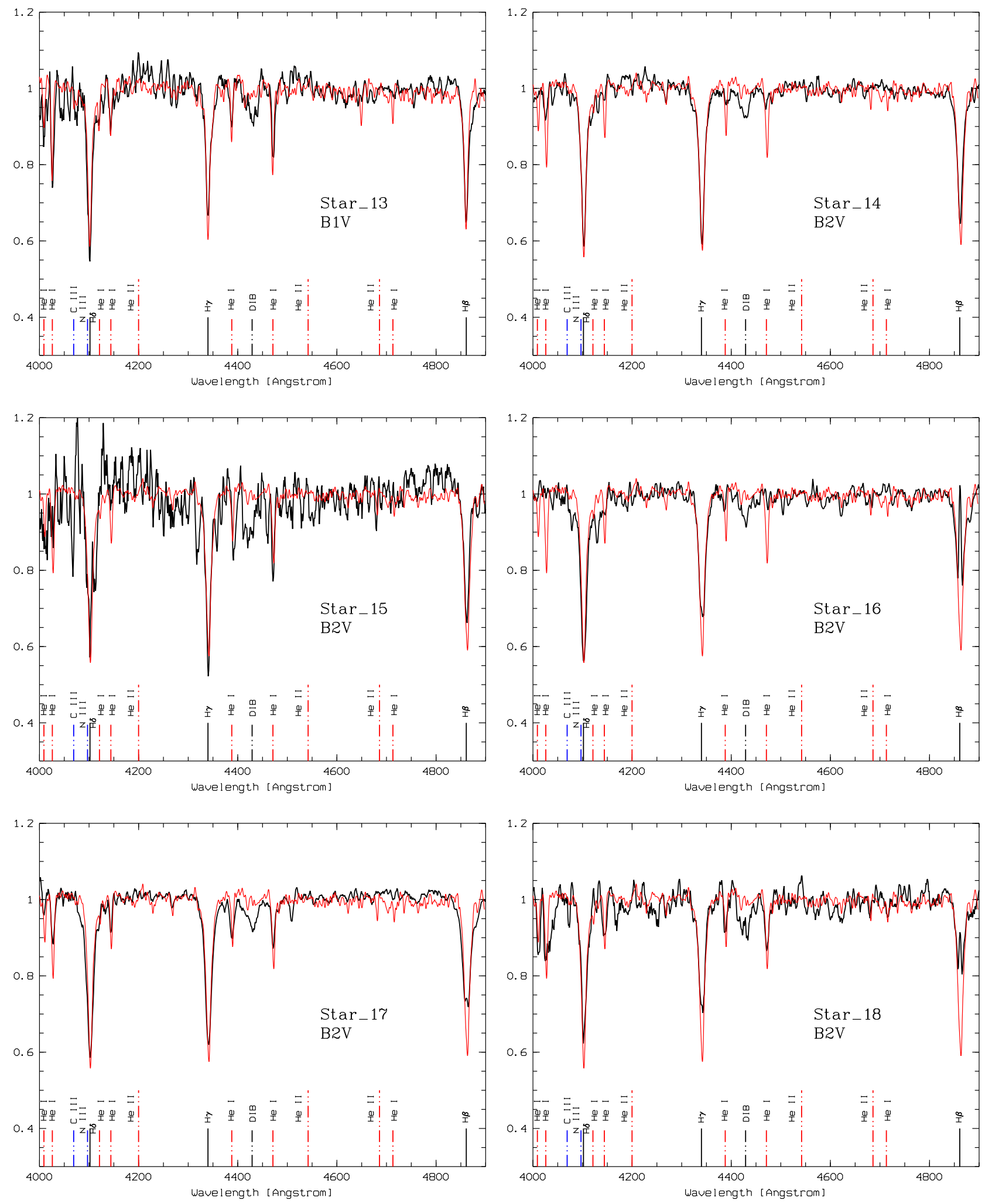

Fig. B.1. continued. 
D. Russeil et al.: NGC 6334 and NGC 6357
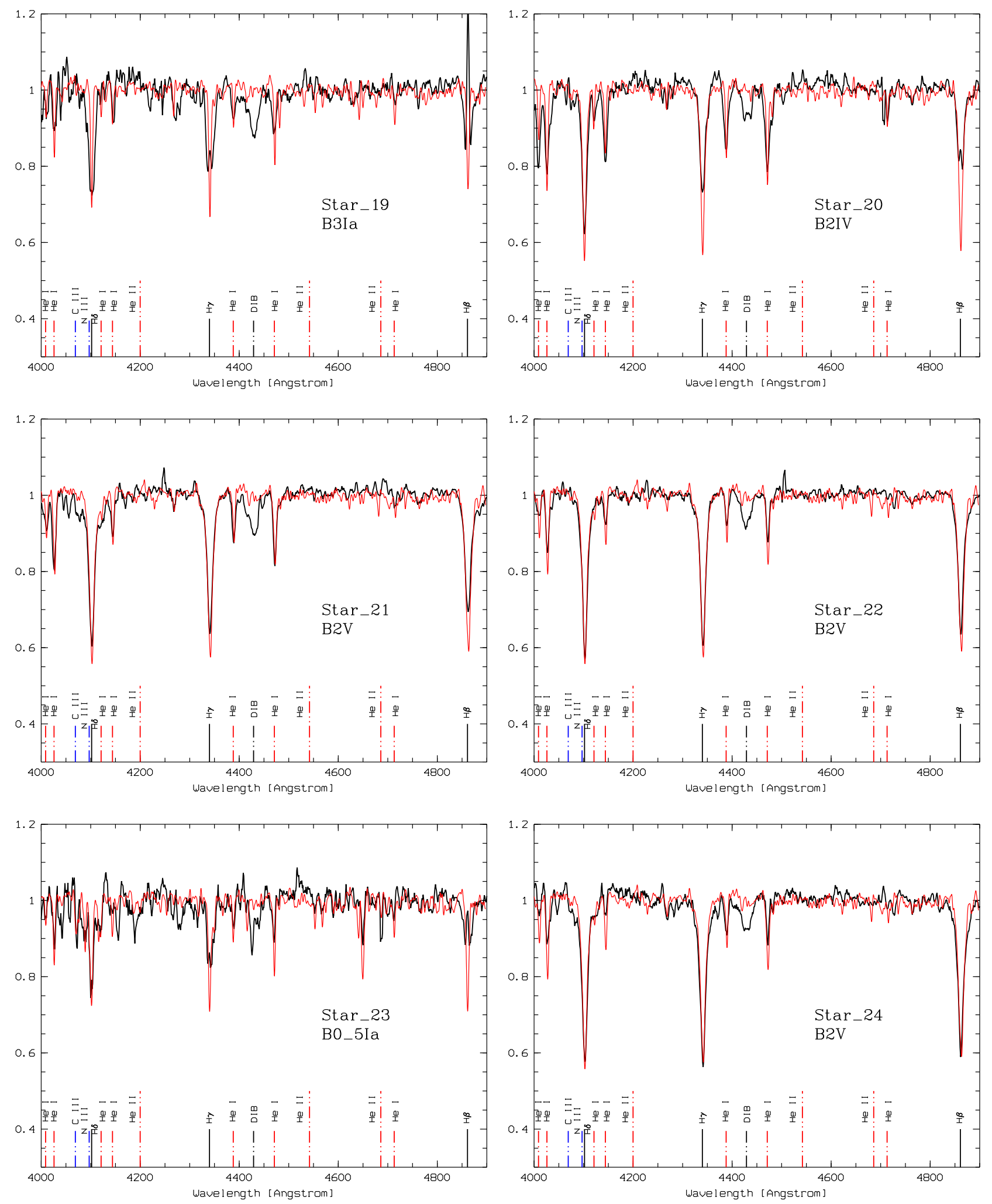

Fig. B.1. continued. 

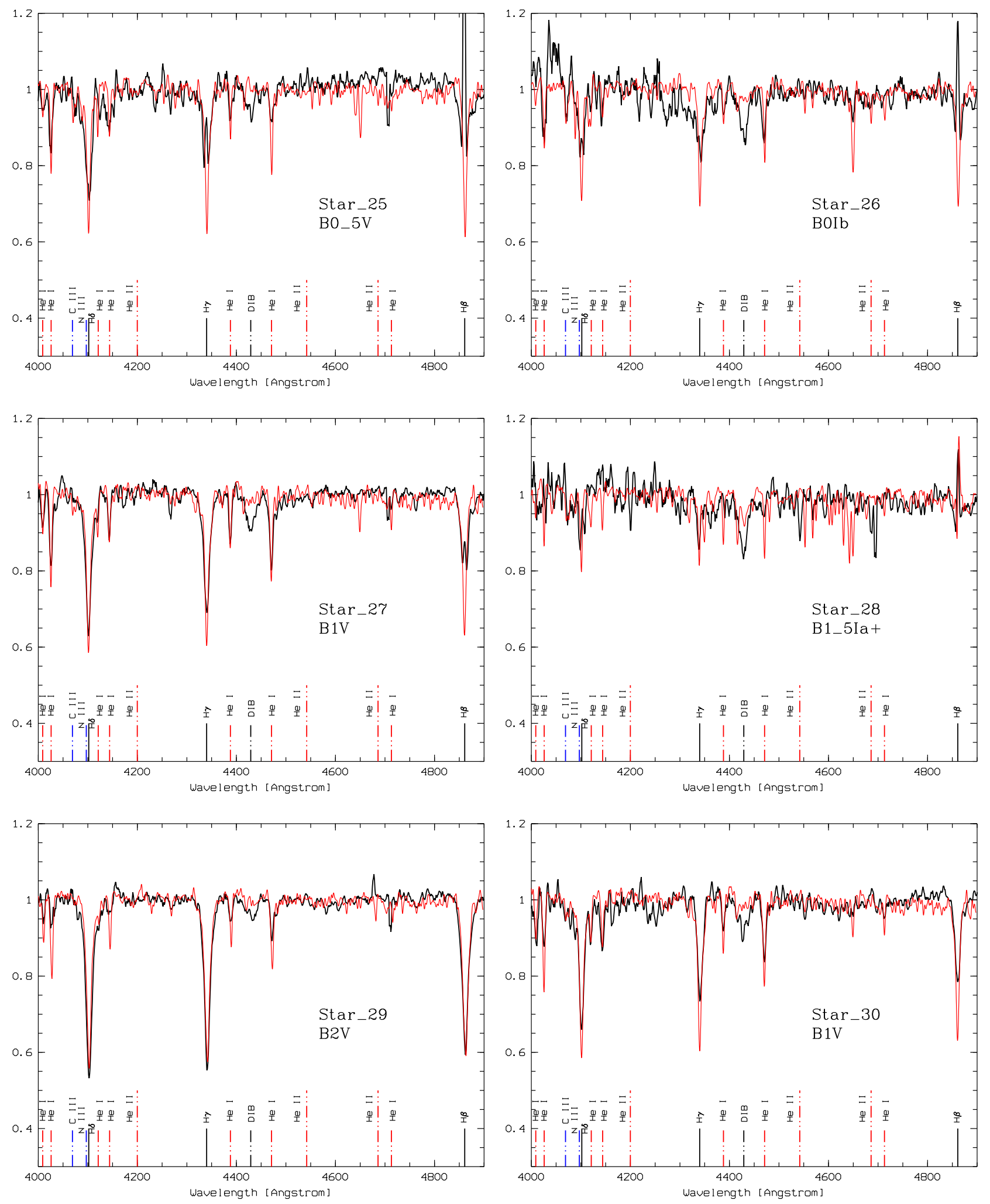

Fig. B.1. continued. 
D. Russeil et al.: NGC 6334 and NGC 6357
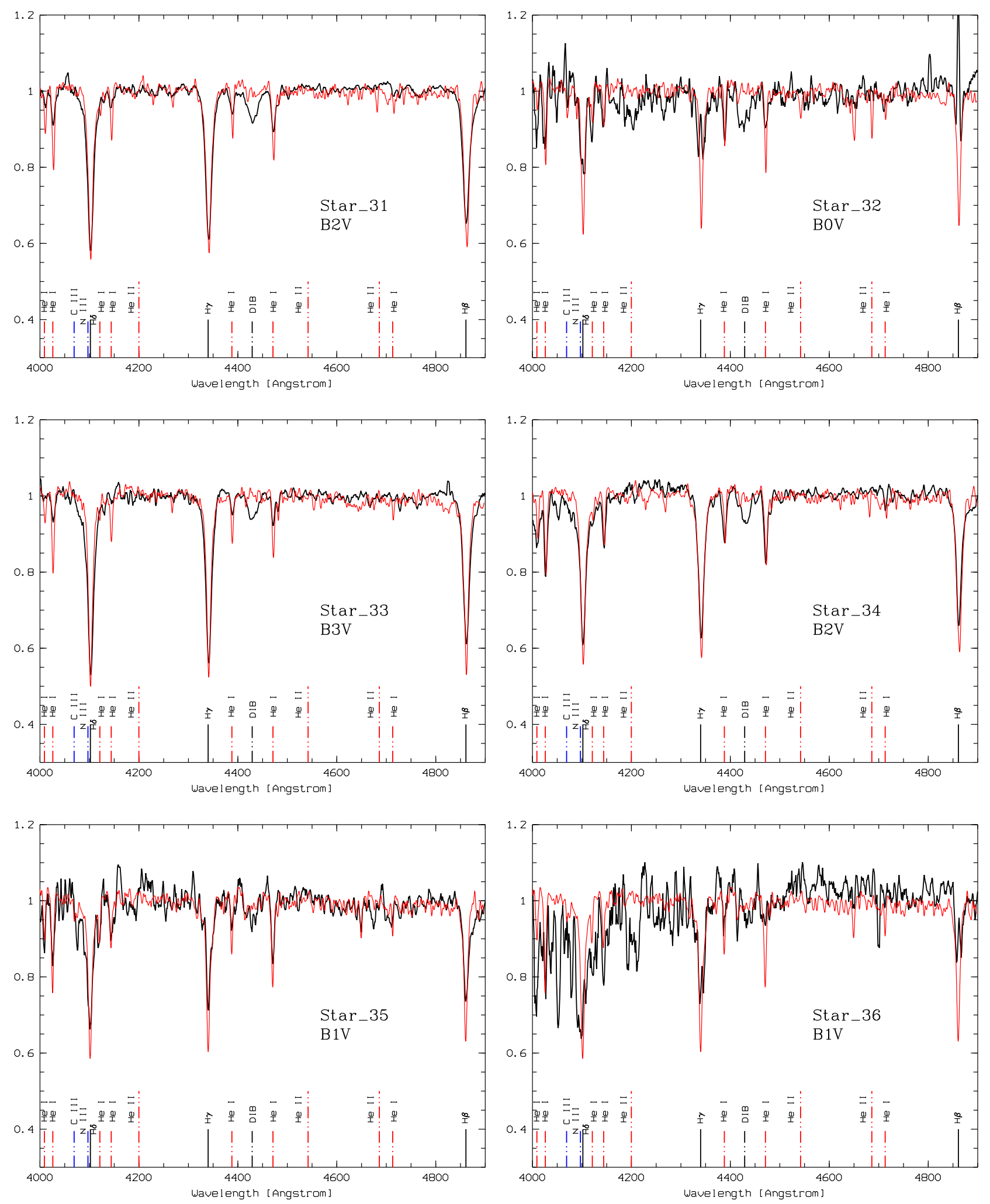

Fig. B.1. continued. 

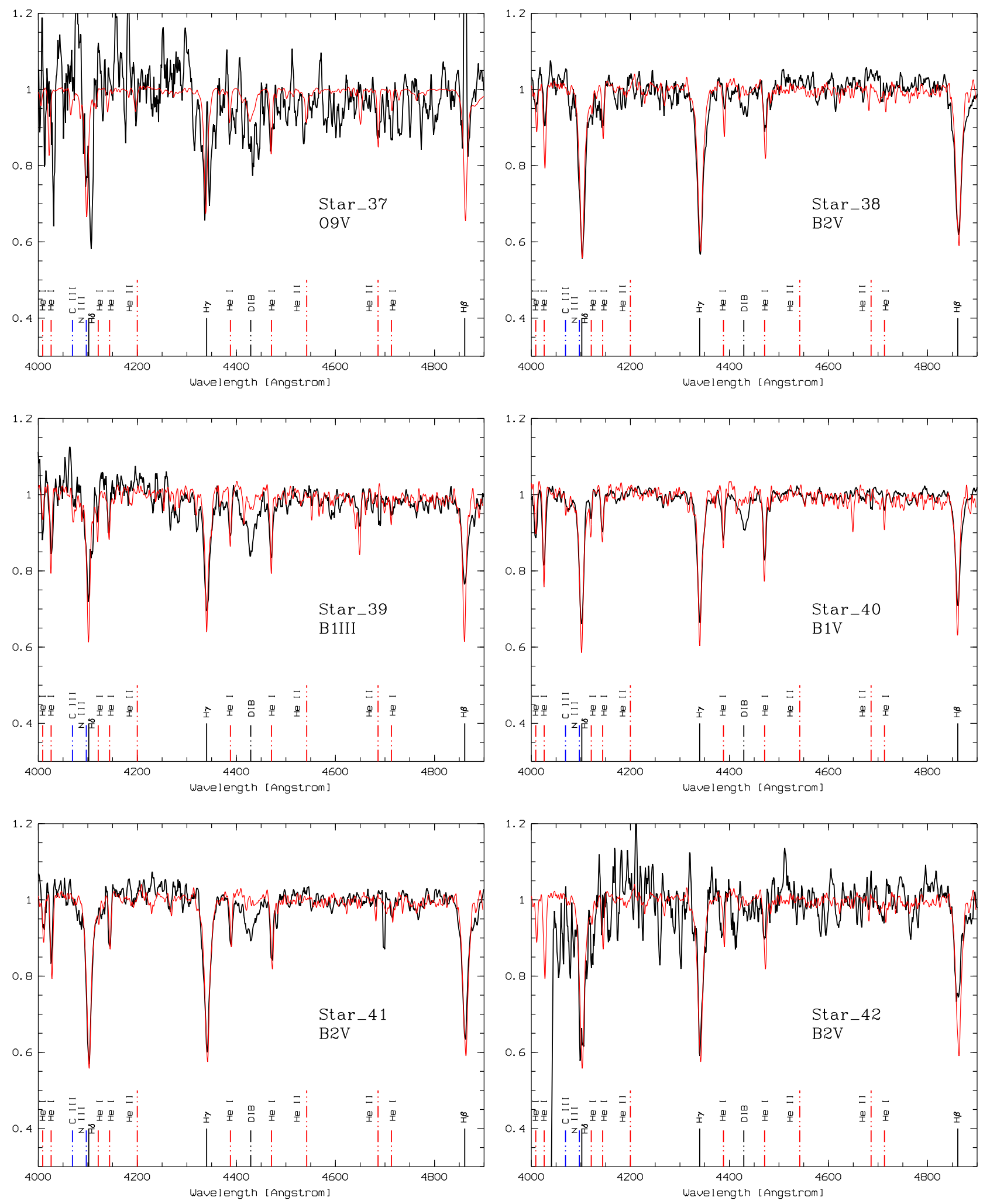

Fig. B.1. continued. 
D. Russeil et al.: NGC 6334 and NGC 6357
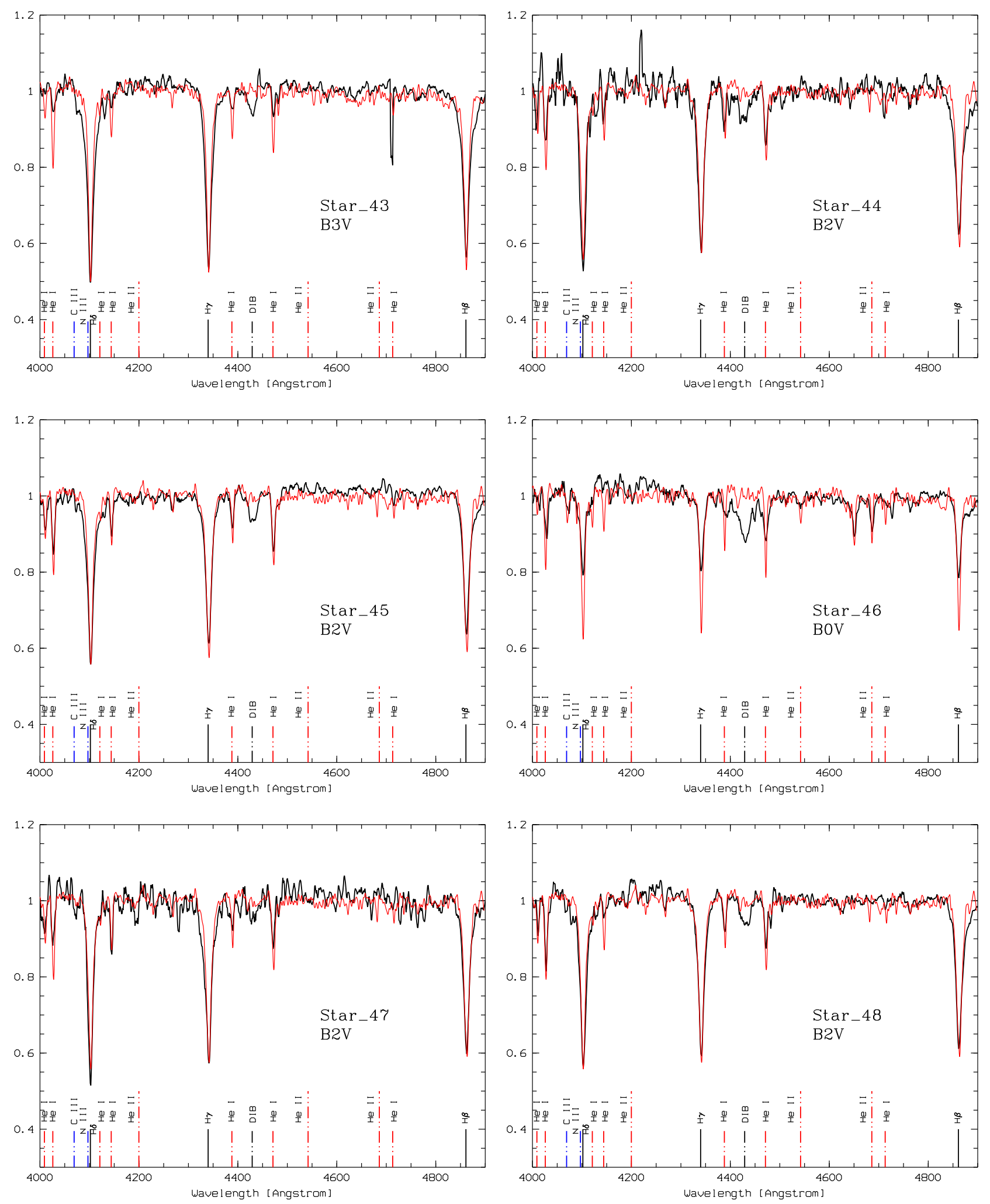

Fig. B.1. continued. 

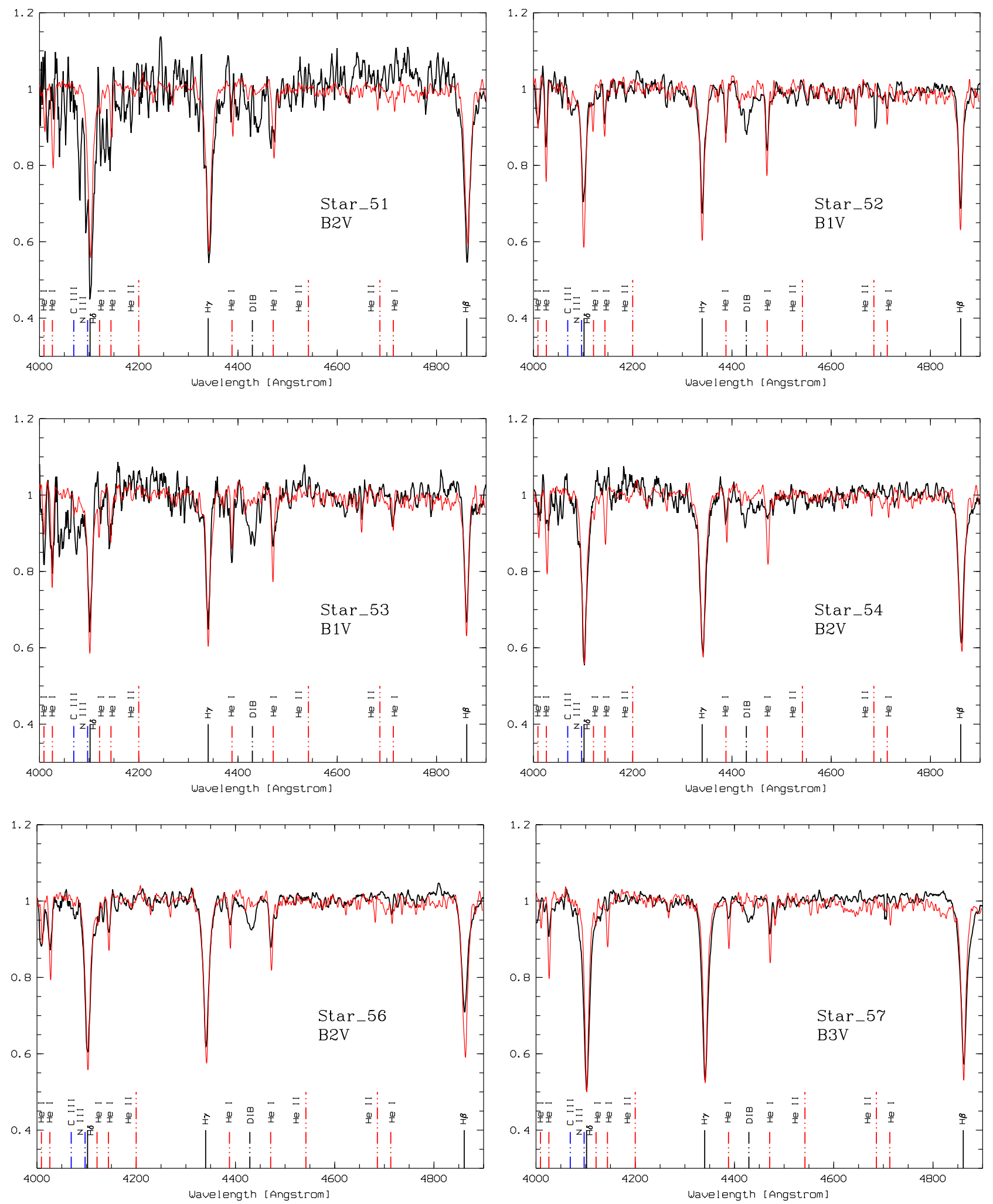

Fig. B.1. continued. 

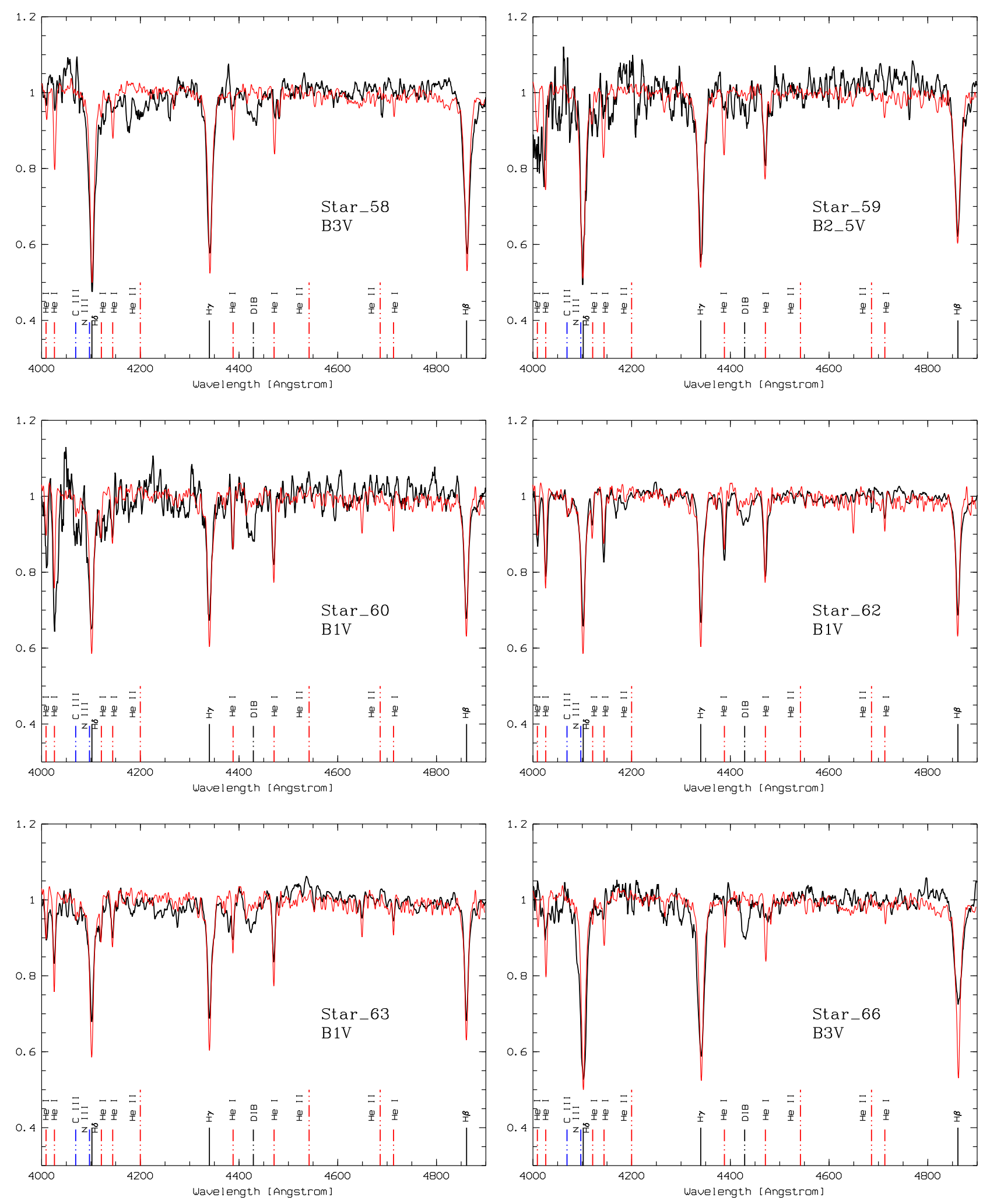

Fig. B.1. continued. 

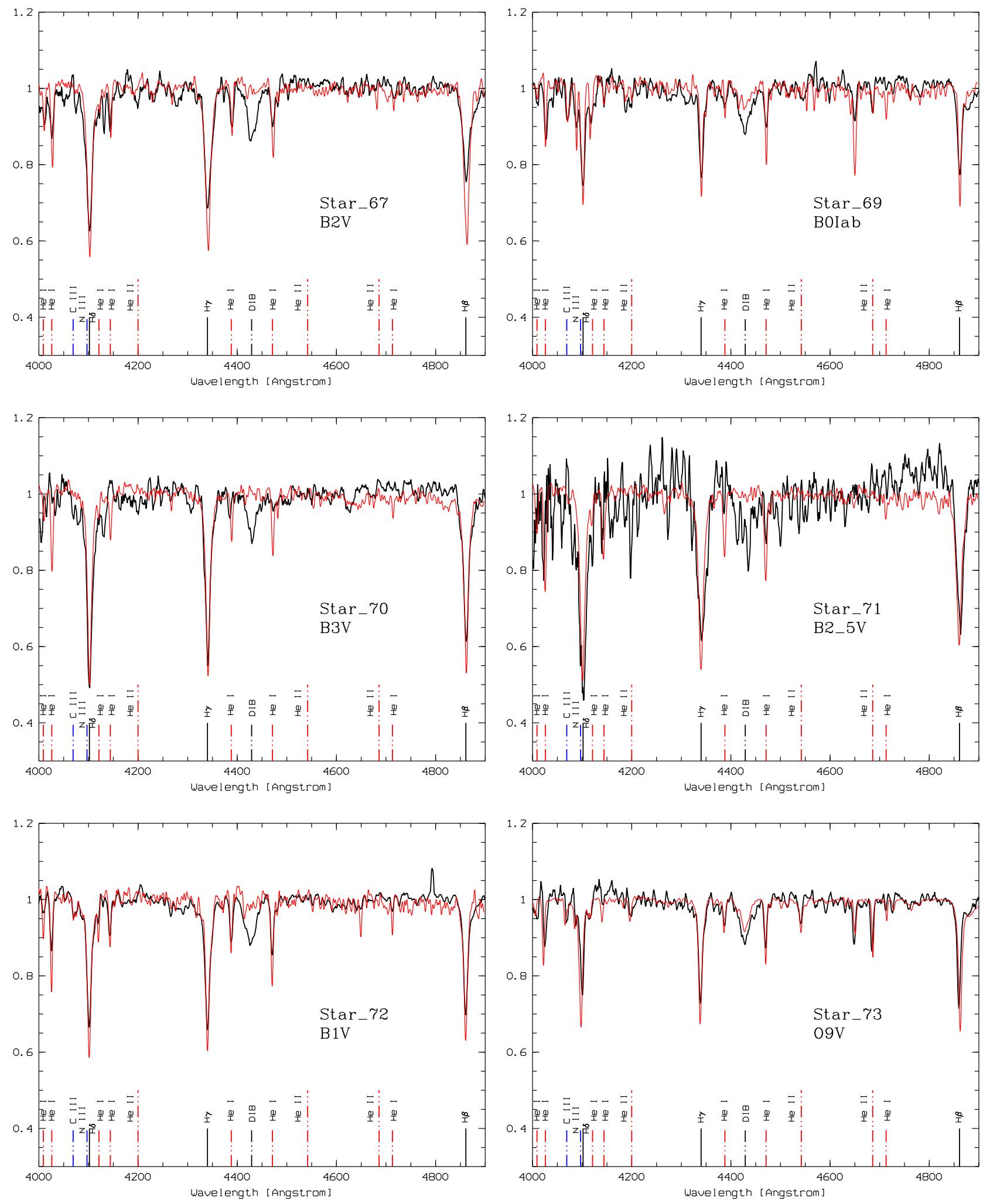

Fig. B.1. continued. 

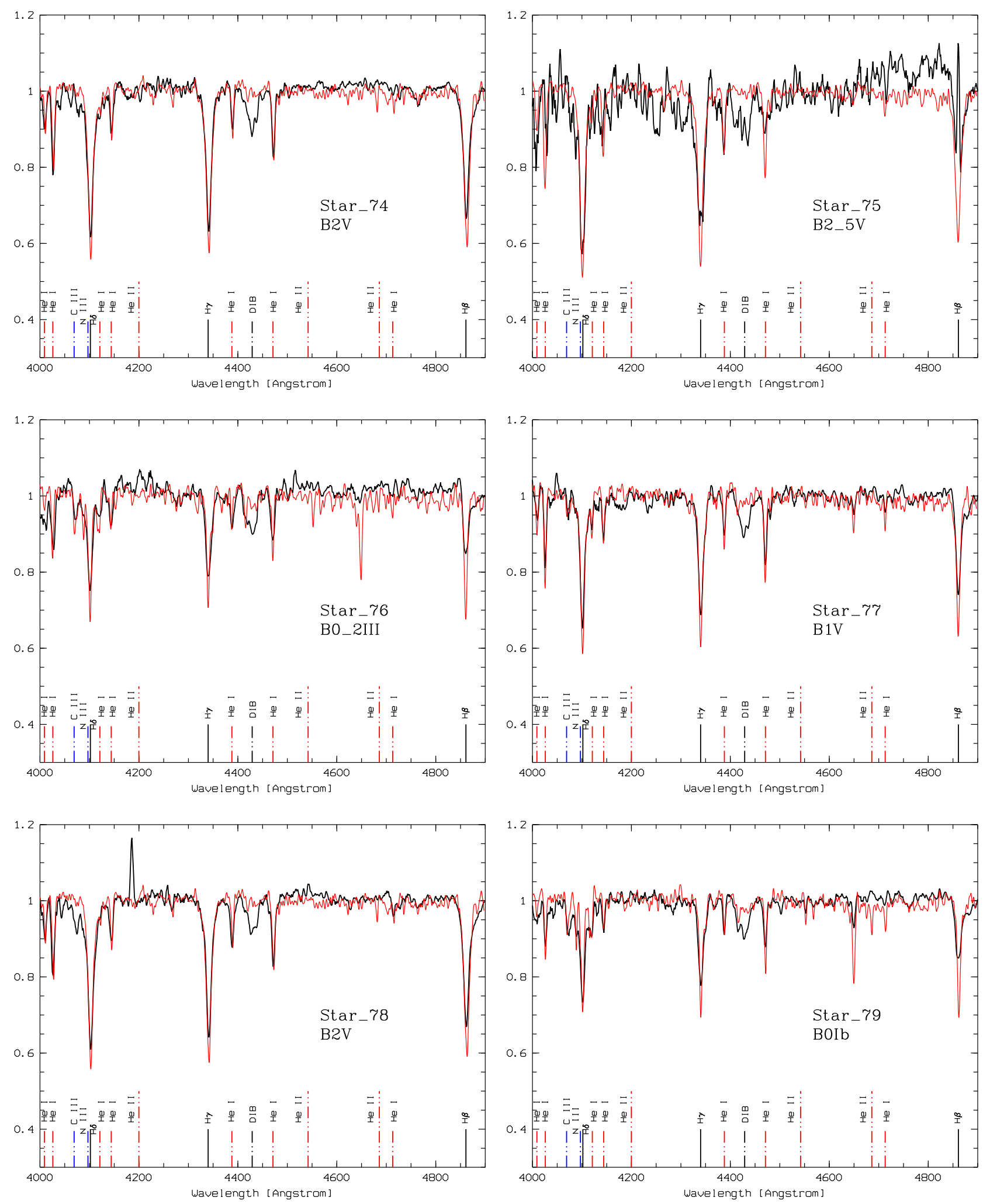

Fig. B.1. continued. 

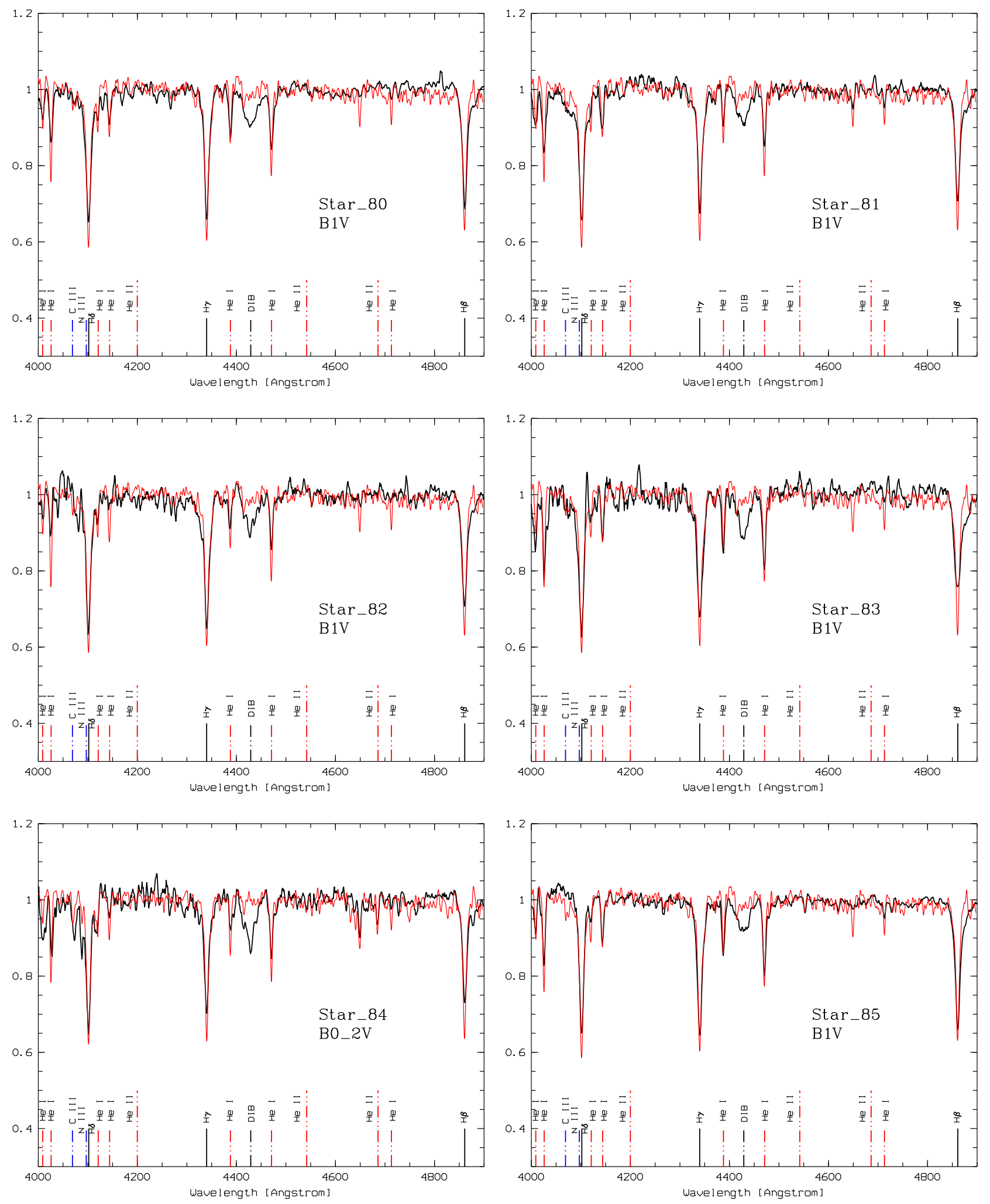

Fig. B.1. continued. 
D. Russeil et al.: NGC 6334 and NGC 6357
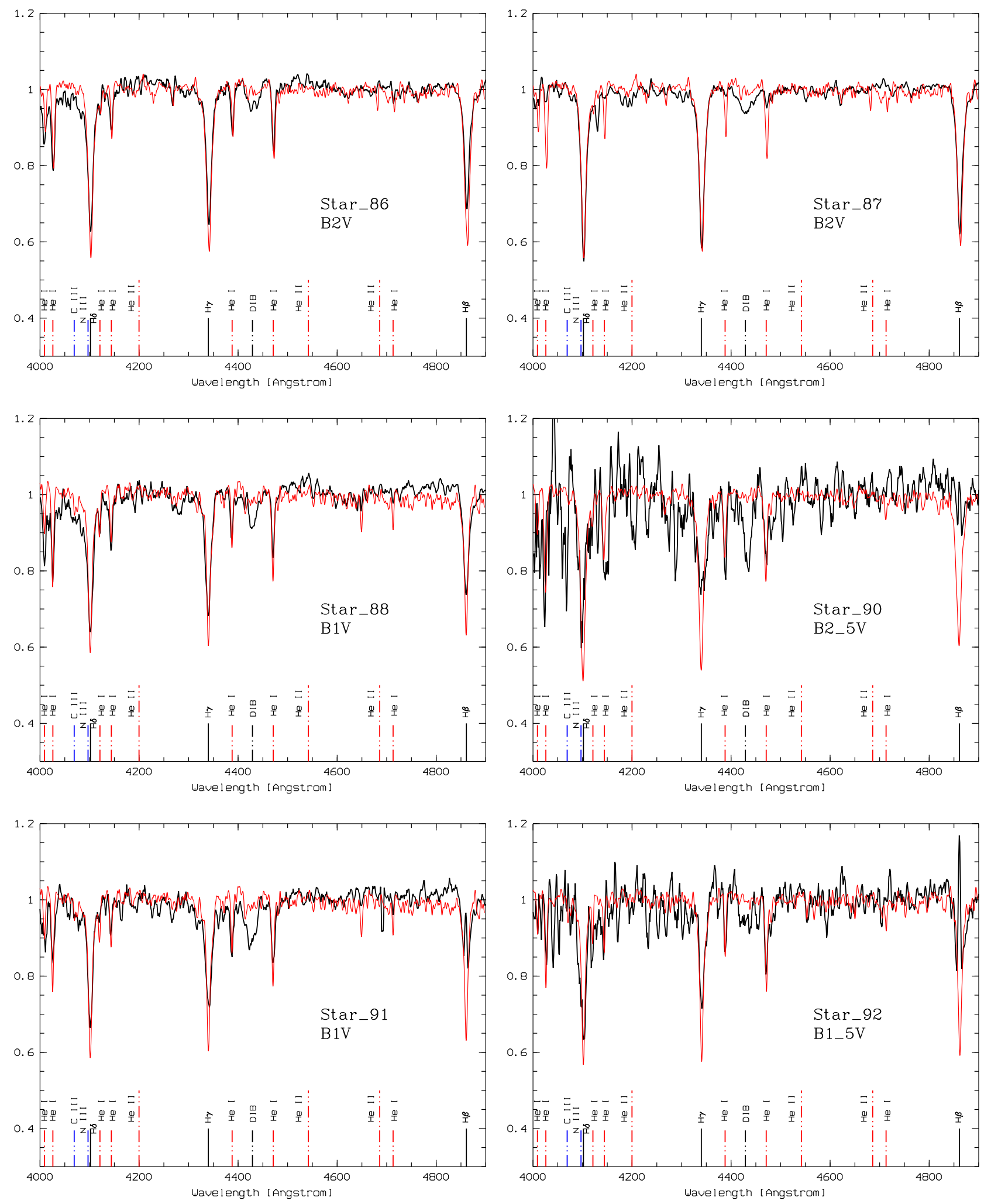

Fig. B.1. continued. 

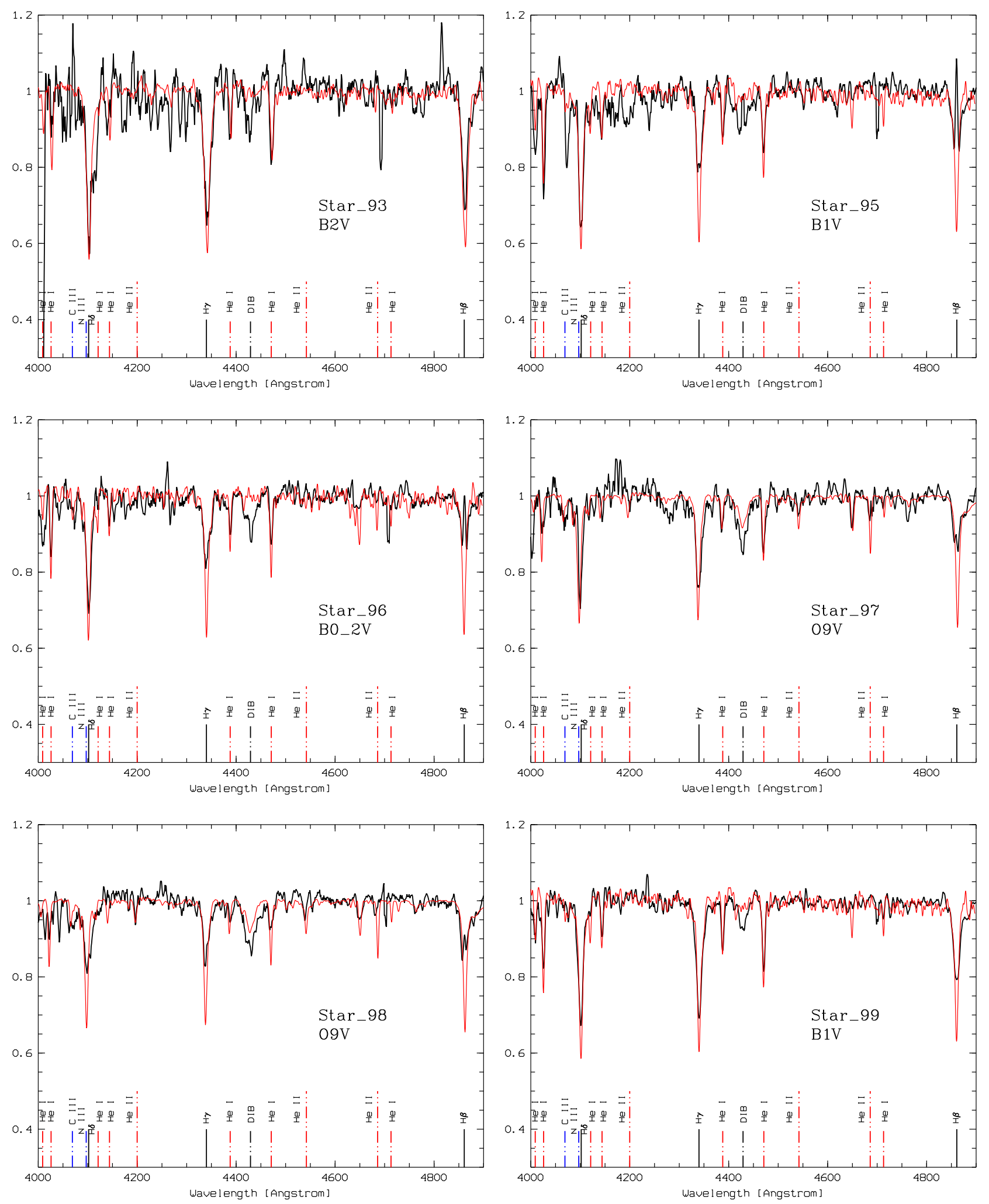

Fig. B.1. continued. 
D. Russeil et al.: NGC 6334 and NGC 6357
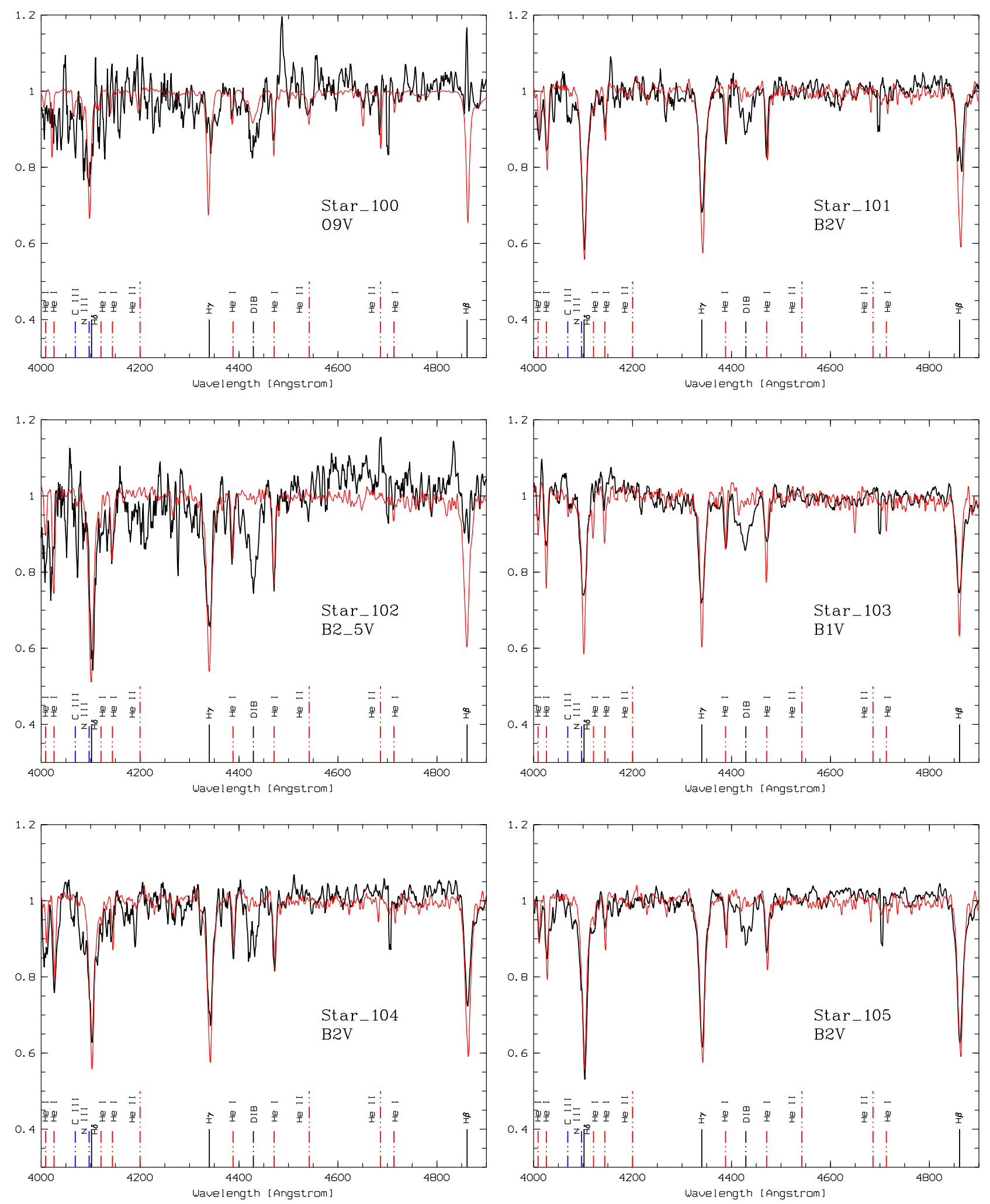

Fig. B.1. continued. 
A\&A 607, A86 (2017)
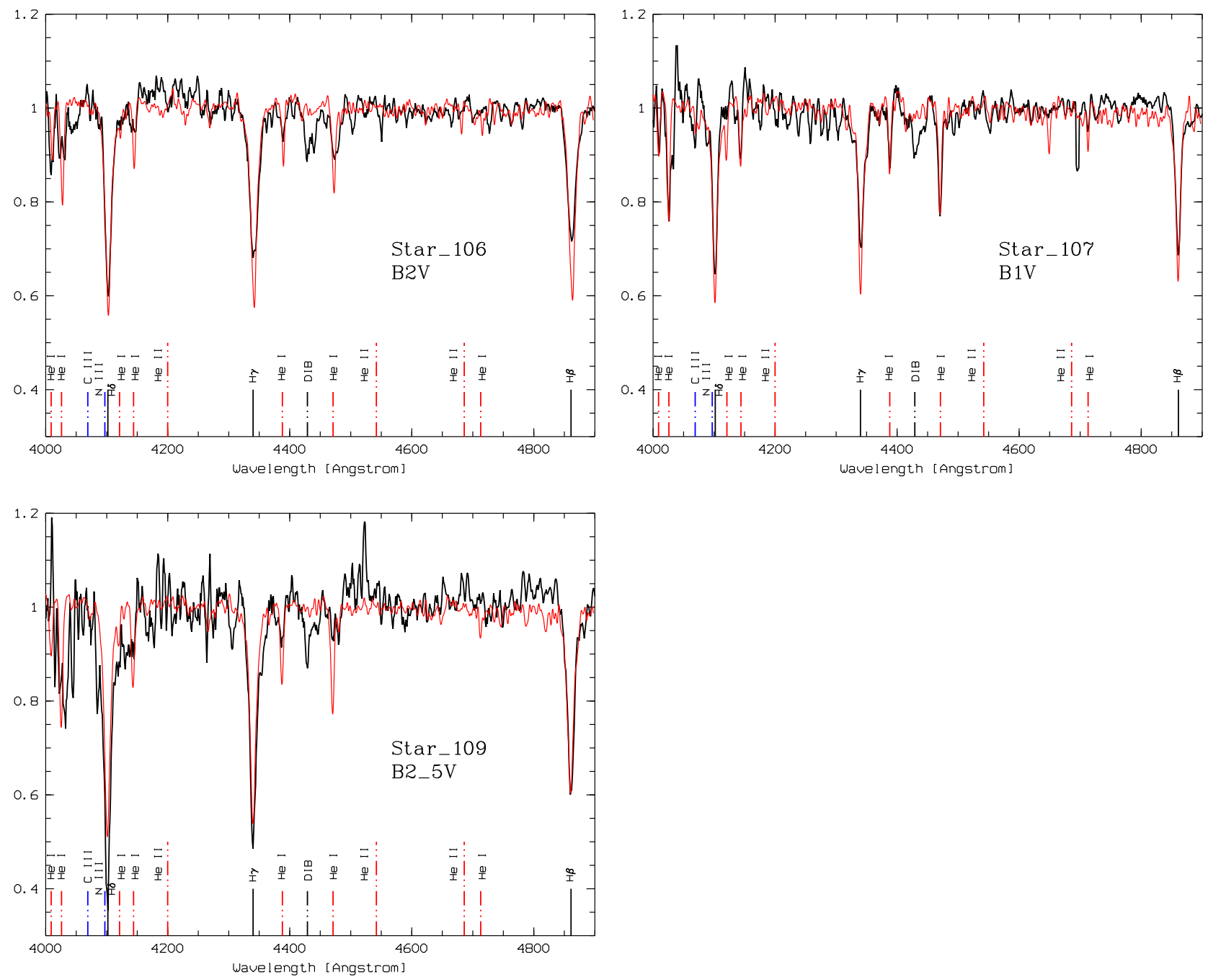

Fig. B.1. continued. 